\title{
NGC 1266: Characterization of the Nuclear Molecular Gas in an Unusual SB0 Galaxy
}

\author{
J. Glenn, N. Rangwala, P.R. Maloney, \& J. R. Kamenetzky \\ CASA, 389-UCB, Department of Astrophysical and Planetary Sciences, University of \\ Colorado, Boulder, CO, 80309 \\ jason.glenn@colorado.edu
}

Received —

Accepted by the Astrophysical Journal on January 13, 2015 


\begin{abstract}
With a substantial nuclear molecular gas reservoir and broad, high-velocity CO molecular line wings previously interpreted as an outflow, NGC 1266 is a rare SB0 galaxy. Previous analyses of interferometry, spectrally resolved low- $J$ CO emission lines, and unresolved high- $J$ emission lines have established basic properties of the molecular gas and the likely presence of an AGN. Here, new spectrally resolved CO $J=5-4$ to $J=8-7$ lines from Herschel Space Observatory HIFI observations are combined with ground-based observations and high-J Herschel SPIRE observations to decompose the nuclear and putative outflow velocity components and to model the molecular gas to quantify its properties. Details of the modeling and results are described, with comparisons to previous results and exploration of the implications for the gas excitation mechanisms. Among the findings, like for other galaxies, the nuclear and putative outflow molecular gas are well represented by components that are cool $\left(T_{\text {nuclear }}=6_{-2}^{+10} \mathrm{~K}\right.$ and $\left.T_{\text {outflow }} \sim 30 \mathrm{~K}\right)$, comprising bulk of the mass (Log $M_{\text {nuclear }} / M_{\odot}=8.3_{-0.4}^{+0.5}$ and $\left.\log M_{\text {outflow }} / M_{\odot}=7.6_{-0.3}^{+0.3}\right)$, and the minority of the luminosity $\left(\log L_{\text {nuclear }} / L_{\odot}=5.44_{-0.18}^{+0.22}\right.$ and $\left.\log L_{\text {outflow }} / L_{\odot} \sim 6.5\right)$ and $\mathrm{warm}$ $\left(T_{\text {nuclear }}=74_{-26}^{+130} \mathrm{~K}\right.$ and $\left.T_{\text {outflow }}>100 \mathrm{~K}\right)$, comprising a minority of the mass $\left(\log M_{\text {nuclear }} / M_{\odot}=7.3_{-0.5}^{+0.5}\right.$ and $\left.\log M_{\text {outflow }} / M_{\odot} \sim 6.3\right)$ but the majority of the luminosity $\left(\log L_{\text {nuclear }} / L_{\odot}=6.90_{-0.16}^{+0.16}\right.$ and $\left.\log L_{\text {outflow }} / L_{\odot} \sim 7.2\right)$. The outflow has an anomalously high $L_{\mathrm{CO}} / L_{\mathrm{FIR}}$ of $1.7 \times 10^{-3}$ and is almost certainly shock excited.
\end{abstract}

Subject headings: Galaxies: ISM - Galaxies: active — Galaxies: jets — Galaxies: individual(NGC 1266) — ISM: molecules — Submillimeter: galaxies 


\section{Introduction}

Massive galaxies with unusual properties can yield insight into short-lived (and perhaps episodic) stages in galaxy evolution. The relatively nearby galaxy NGC1266 1 has been classified variously as an S0, SB0, SA, and a LINER galaxy. It is unusual in that it contains a large quantity of nuclear molecular gas and a high-velocity molecular line component: Alatalo et al. (2011) discovered a $1.1 \times 10^{9} \mathrm{M}_{\odot}$ centrally concentrated (60 pc radius) molecular gas mass and what they interpret as a $2.4 \times 10^{7} \mathrm{M}_{\odot}$ molecular outflow extending some $460 \mathrm{pc}$ in radius, with emission extending to $\pm 400 \mathrm{~km} \mathrm{~s}^{-1}$ and an outflow rate of 13 $\mathrm{M}_{\odot} \mathrm{yr}^{-1}$. The nuclear molecular gas concentration is potentially an indication of a merger event, although no merger partners are apparent. Because the mass outflow rate exceeds the star formation rate inferred from the far-IR luminosity, Alatalo et al. (2011) surmised that an AGN powers the molecular outflow. Although only weak hard X-ray emission was detected by Chandra X-ray Observatory, it is possible that the AGN is buried beneath Compton-thick absorption. Nyland et al. (2013) presented VLBA observations indicating a very high brightness temperature $\left(>1.5 \times 10^{7} \mathrm{~K}\right)$ radio continuum emission $1.2 \mathrm{pc}$ in size, coincident with the densest molecular gas, and JVLA HI absorption observations consistent with the outflow. Observations of atomic line emission indicated velocities reaching \pm 900 $\mathrm{km} \mathrm{s}^{-1}$, the presence of shocked gas, and a morphology consistent with "nascent" radio jets (Davis et al. 2012). It is possible that the molecular high-velocity gas is an outflow and is associated with the ionized gas, but it could also be in a rapidly rotating disk. Integral field spectroscopy and Swift UV/optical observations indicate central-parsec stellar populations $<1$ Gyr old and a post-starburst spectrum (Alatalo et al. 2014).

Recently, Pellegrini et al. (2013) analyzed ground-based and Herschel Space

\footnotetext{
${ }^{1} z=0.0072$, distance $=30.06 \mathrm{Mpc}($ Temi et al. 2009); M $=-22.93$, half-light radius 20.4" (Cappellari et al. 2011); $\log L_{\mathrm{FIR}}\left(\mathrm{L}_{\odot}\right)=10.44($ Kamenetzky et al. 2014)
} 
Observatory SPIRE observations of CO from $J=1-0$ to $J=13-12$ and $\mathrm{H}_{2} \mathrm{O}$ lines. They concluded that the far-infrared spectrum was ULIRG-like and, based on comparisons to photon-dominated regions and shock models, that the gas is likely shock heated, although they could not spectrally resolve the CO lines with SPIRE to separate the disk and outflow components. Determining the physical state of the molecular gas is crucial to understanding its excitation mechanism(s), and determining the masses of the centrally concentrated gas and the outflow are necessary to determine the lifetime of this evolutionary phase, how much gas is available for star formation, and what the fate of the gas will be. Here, we analyze new Herschel HIFI observations of CO up to $J=8-7$ that spectroscopically resolve the outflow and centrally concentrated disk components, and reanalyze Herschel SPIRE FTS observations up to $J=13-12$ in combination with the HIFI observations to quantify the physical parameters of these gas components. In $\S 2$, we present the Herschel HIFI and SPIRE observations. In $\S 3$ we present dust modeling and in $\S 4$ non-LTE models of the $\mathrm{CO}$ emission that constrain the gas physical conditions. In $\S 5$ we compare to previous results and comment on the possible excitation mechanisms of the gas. Our conclusions are summarized in $\S 6$.

\section{Observations and Line Fitting}

Four sets of observations were utilized for this analysis: (1) Herschel SPIRE Fourier Transform Spectrometer (FTS) spectroscopy of CO from $J=4-3$ to $J=13-12$ and SPIRE $250 \mu \mathrm{m}, 350 \mu \mathrm{m}$, and $500 \mu \mathrm{m}$ dust continuum photometry, (2) Herschel HIFI observations of the $J=5-4,6-5,7-6$, and $8-7$ lines, (3) ground-based low $-J \mathrm{CO}$ observations from the literature, and (4) continuum photometry from the literature. The SPIRE FTS (Griffin et al. 2010) provided continuous spectral coverage from $450 \mathrm{GHz}$ to 1,600 GHz with a spectral resolution of $1.44 \mathrm{GHz}$. Bands 1b, 2a, 3a, and 3b of the HIFI 
receivers (de Graauw et al. 2010) on board Herschel (Pilbratt et al. 2010) were used in single-point Wide Band Spectrometer mode. All the Herschel data were reduced with HIPE-9. Both sets of Herschel observations are listed in Table 1 and the FTS spectrum is shown in Figure 1. The dust continuum emission, with flux density rising with frequency, and bright $\mathrm{CO}$ and $\mathrm{H}_{2} \mathrm{O}$ water lines are apparent, as are the $\mathrm{CI} J=2-1$ and [NII] lines. The line fluxes reported in Table 2 were derived by fitting sinc functions (the intrinsic instrumental line profile, as the lines are unresolved). The HIFI spectra are shown in Figure 2. For two of the lines $(J=5-4$ and $J=6-5)$ the $\mathrm{V}$ and $\mathrm{H}$ senses of polarization were combined. For the other two, only one sense was included because the baselines were poor for the orthogonal polarizations. The ground-based CO lines $(J=3-2,2-1$, and $1-0)$ were taken from Alatalo et al. (2011).

Following Alatalo et al. (2011), the CO lines were fit with double Gaussian functions to account for the broad (outflow) component (hereafter Broad) and the narrow, central velocity component (hereafter CVC), presumably a disk, and flat baselines. The FWHM of the CVC was fixed at $123 \mathrm{~km} / \mathrm{s}$ based on fits to the $J=2-1(\mathrm{FWHM}=123.9 \pm 1.5 \mathrm{~km}$ $\left.\mathrm{s}^{-1}\right)$ and $J=3-2$ lines $\left(\mathrm{FWHM}=122.2 \pm 1.7 \mathrm{~km} \mathrm{~s}^{-1}\right)$, which had the highest $\mathrm{S} / \mathrm{N}$. The Broad component line widths were allowed to vary, and for all but the $J=1-0$ line (for which the Broad component appears very weak), the line widths are consistent with 278 $\mathrm{km} \mathrm{s}^{-1}$ within $1 \sigma$ (with the exception of $1.4 \sigma$ in the case of the $\mathrm{J}=5-4$ line and with $1 \sigma$ error bars in excess of $100 \mathrm{~km} \mathrm{~s}^{-1}$ for the $\mathrm{J}=7-6$ and $8-7$ lines). We chose to fix the CVC line width (and not the Broad component line width) because it is much more plausible that the disk component has a line width independent of $J$ than the outflow.

We compared the reduced $\chi^{2}$ between single Gaussian and double Gaussian fits and found clear evidence for outflow (Broad component) in the CO $J=5-4$ and $J=6-5$ lines. The $\mathrm{S} / \mathrm{N}$ in the cases of the $\mathrm{CO} J=7-6$ and $J=8-7$ was not high enough 
to unambiguously distinguish the Broad and CVC components, i.e., the reduced $\chi^{2}$ was the same for single and double Gaussian fits. We report the line widths and integrated line fluxes for the Broad component and CVC in Table 3. For comparison, Alatalo et

al. (2011) measured CVC line widths of 114 to $134 \mathrm{~km} \mathrm{~s}^{-1}$ for $J=1-0$ to $J=3-2$ (with uncertainties up to a few $\mathrm{km} \mathrm{s}^{-1}$ ) and adopted a single line width of $353 \pm 17$ $\mathrm{km} \mathrm{s}^{-1}$ for the Broad component from a fit to the $J=2-1$ line. Our CVC width is comparable, but our Broad component linewidth differs by almost $4 \sigma$; it is possible that the discrepancy arises from different treatment of the baselines. The lines fluxes were converted from $\mathrm{K}$ to Jy km/s using the conversion factors given in the HIFI Observers' Manual (herschel.esac.esa.int/Docs/HIFI/pdf/hifi_om.pdf, p. 73, Table 5.5). We assumed that the molecular emission region in NGC 1266 is compact compared to Herschel's beams, which is supported by Alatalo et al. (2011).

\section{Dust Modeling}

The dust emission was modeled both to obtain the dust properties (luminosity, temperature, and mass) and to derive the wavelength-dependent dust optical depth for an extinction correction to the high $-J$ CO lines. The dust parameters were fit using the nested sampling algorithm PyMultiNest (Feroz and Hobson 2008; Feroz et al. 2009; Feroz et al. 2013; Buchner et al. 2014) with the method presented in Kamenetzky et al. (2014; hereafter K14); the photometric data are in Table 4. We adopt the Casey (2012) model which sums a grey-body distribution and a mid-infrared power-law distribution with an exponential cutoff to account for warm dust. The free parameters are $T$ (temperature, K), $\beta$ (emissivity index), $\lambda_{0}$ (wavelength at which optical depth is unity, $\mu \mathrm{m}$ ), and $\alpha$ (power-law index). We assumed that calibration errors were $50 \%$ correlated between measurements in different bands of the same instruments (neither $0 \%$ nor $100 \%$ were appropriate and 
experimentation showed that the parameters were not highly sensitive to intermediate assumptions).

The dust-fitting results and best-fit to the dust spectral energy distribution are given in Table 5 and shown in Figure 3, respectively, and the likelihood distributions for the fitted parameters are in Figure 4. The dust is warm, with a small temperature uncertainty, $56 \pm 3$ $\mathrm{K}$, although the uncertainties in $\lambda_{0}$ and $\beta$ are larger $\left(\sigma_{\lambda_{0}}=25 \mu \mathrm{m}\right.$ and $\left.\sigma_{\beta}=0.29\right)$ and these are what most affect the CO extinction correction (see Section 4). The luminosity, Log

$L_{\text {dust }} / L_{\odot}=10.44 \pm 0.01$, is well constrained. Using a dust opacity of $\kappa_{125 \mu \mathrm{m}}=2.64 \mathrm{~m}^{2} \mathrm{~kg}^{-1}$ (Dunne et al. 2003), the derived dust mass is $\log M_{\text {dust }} / M_{\odot}=6.34 \pm 0.04$, corresponding to $\log M_{\text {gas }} / M_{\odot}=8.34 \pm 0.04$ for a gas-to-dust mass conversion factor of 100 . The quoted uncertainties account only for the statistical uncertainties in the derived gas mass. The dust opacity is probably uncertain to a factor of approximately two, which propagates to an uncertainty of 0.3 dex in the dust mass. Furthermore, the dust-to-gas mass conversion factor is probably uncertain up to a factor of two, yielding a net systematic uncertainty of $\sim 0.5$ dex in the derived gas mass, dominating over the statistical uncertainty.

\section{Non-LTE CO Modeling and Gas Parameters}

To determine the gas physical conditions, we used a custom version of the RADEX non-LTE code (van der Tak et al. 2007) combined with a Bayesian analysis (see Rangwala et al. 2011, Scott et al. 2011, Kamenetzky et al. 2012, and Kamenetzky et al. 2014). RADEX computes the intensities of molecular lines by iteratively solving for statistical equilibrium using an escape probability formalism. The inputs to RADEX are the gas density $\left(n_{H_{2}} ; \mathrm{H}_{2}\right.$ is the main collision partner for $\left.\mathrm{CO}\right)$, the kinetic temperature $\left(T_{k i n}\right)$, and the CO column density per unit line width $\left(N_{C O} / \Delta v\right)$, which sets the optical depth scale. We also vary the area filling factor $\left(\Phi_{A}\right)$. The code generates a grid of model CO spectral 
line energy distributions (in background-subtracted, Rayleigh-Jeans antenna temperatures) for a large range of input parameters. From the marginalized best-fit parameters (again using PyMultiNest), we calculate the likelihoods of a set of secondary parameters, such as pressure and molecular gas mass.

Generally two temperature components are required to fit the spectral line energy distributions; see the discussion in K14. Motivated by the empirical result that the ratio of mass of the warm gas component to the cool gas component traced by $\mathrm{CO}$ in seventeen infrared-luminous nearby galaxies is $0.11 \pm 0.02$ (including NGC 1266, but the results are essentially unchanged if NGC 1266 is excluded; K14), the mass of the warm component was required to be less than the mass of the cool component. Based on interferometry from Alatalo et al. (2011), a source size of $22^{\prime \prime}$ was assumed for the CVC and Broad emitting regions (this constraint is revisited in $\S 5$ ), with areal filling factors $\leq 1$, which allows the sources to take on any sizes up to $22^{\prime \prime}$ to find the model parameters that best represent the data. A CO-to- $\mathrm{H}_{2}$ abundance ratio of $3 \times 10^{-4}$ was assumed to scale the $\mathrm{CO}$ column density to a total molecular gas column density (Lacy et al. 1994; the derived molecular gas masses scale inversely with the assumed value). CO-to- $\mathrm{H}_{2}$ abundance ratios of $1 \times 10^{-4}$ to $3 \times 10^{-4}$ are assumed in the literature. We have chosen the high end of this range, which is as large as the CO abundance can get for gas-phase abundances similar to the Solar neighborhood (most of the gas-phase carbon would be in the form of $\mathrm{CO}$ ), because it makes our mass estimates conservative in the sense of producing minimal molecular gas masses.

We did two types of RADEX model fitting to the CO spectral line energy distributions: (1) using only the lines up to and including $J=8-7$, in which the CVC and Broad components could be distinguished with the spectral resolutions afforded by HIFI and ground-based heterodyne observations, and (2) using the CVC model level populations to generate line fluxes for the $J=9-8$ and higher lines for subtraction from the FTS fluxes 
to estimate and model the Broad component fluxes. Case (2) is included because additional line flux information is available in the FTS spectra: the CVC and Broad components must sum to match the FTS line fluxes (within the photometric uncertainties). The CVC line fluxes, rather than the Broad component fluxes, were subtracted because they were better constrained for more of the low and mid- $J$ lines. To properly treat the uncertainties, the likelihood distributions for the $J=9-8$ and higher lines from the CVC model were used for subtraction from the FTS line fluxes, thereby marginalizing over the ranges of allowed values. We explore Case $(2)$ in the Discussion ( $(5)$ because it makes use of all available information and assumes there are no non-physical discontinuities in the spectral line energy distributions of the different components. Within the two broad categories of models, various additional models were run to test for the effects of extinction and assumptions about multiple components.

We first consider case (1), fitting only up to and including $J=8-7$, for the CVC, with the constraint that the mass of the cold component has to be greater than the mass of the warm component and with no extinction correction applied (Fig. 5 and Table 6). Not surprisingly, given what has been observed for other galaxies, two temperature components are required to simultaneously account for the lowest $-J$ lines $(1-0$ and $2-1)$ and the mid $-J$ lines 2 . The cold component is quite cold, $6_{-1}^{+11} \mathrm{~K}$ (error bars are $\pm 1 \sigma$ unless otherwise

\footnotetext{
${ }^{2}$ Although only two components are required to fit the spectral line energy distribution well, it is likely that there are a continuum of temperatures and densities in the NGC1266 molecular interstellar medium. K14 has a discussion of the numbers of components. Further work exploring, e.g., models with power-law distributions of temperature, density, and column density is needed. For the time being, we acknowledge that a continuum of physical conditions is likely present but also conclude that a cold component is required to explain the low $-J$ line fluxes and a higher-excitation component is required to explain the mid- $J$
} 
indicated, where $\pm 1 \sigma$ encompasses $68 \%$ of the likelihood distributions about the means), and the warm component is $74_{-26}^{+134} \mathrm{~K}$. The densities of the two components are not well constrained, both with appreciable likelihood from $10^{2}$ to $10^{7} \mathrm{~cm}^{-3}$. Because the density likelihoods are so broad, the pressures (the products of the temperatures and densities) are poorly constrained 3 For the cold component the pressure likelihood is appreciable from $10^{3}$ to $10^{8} \mathrm{~K} \mathrm{~cm}^{-3}$. The pressure of the warm component has a median of $10^{6.3} \mathrm{~K} \mathrm{~cm}^{-3}$ and a $\pm 1 \sigma$ range of approximately one dex. The luminosities, which are largely independent of the model parameters, are well separated and well constrained, with the cool component $\left(\log L / L_{\odot}=5.44_{-0.18}^{+0.22}\right)$ required to explain the luminosities of the lowest few lines and the warm $\left(\log L / L_{\odot}=6.90_{-0.16}^{+0.16}\right)$ component almost entirely responsible for the luminosities of the lines above $J=5-4$. Only about $3 \%$ of the luminosity emerges in the low $-J$ lines, which is on the low end of the range observed in K14, but not extraordinary. As with density and temperature, column density and beam filling factor are degenerate and anticorrelated in fits; thus, we focus on their product, the beam-averaged column density, which is converted to molecular gas mass as in K14. The cold and warm component masses are $\log M / M_{\odot}=8.3_{-0.4}^{+0.5}$ and $\log M / M_{\odot}=7.3_{-0.5}^{+0.5}$, yielding a cold-to-warm component mass ratio of 0.1 , similar to the average of the K14 sample $(0.11 \pm 0.02)$.

With optically thick dust at far-infrared wavelengths $\left(\tau_{100 \mu \mathrm{m}}=4.7 \pm 1.9\right)$, the high $-J$ CO lines are likely subject to extinction. Under the assumption of well-mixed molecular gas and dust, the extinction correction can be applied by dividing the line fluxes by a factor

lines - a single component cannot simultaneously do both.

${ }^{3}$ Temperature and density are typically degenerate over a fairly broad range in the collisional excitation of $\mathrm{CO}$, although their product, the pressure, is usually better constrained because the temperature and density are anticorrelated in fits: temperatures and densities can trade off to yield similar pressures - low temperatures and high densities, and vice versa. 
$\left(1-e^{-\tau_{\lambda}}\right) / \tau_{\lambda}$. The correction has the effect of slightly enhancing the bump in the spectral line energy distribution starting at $J=4-3$ and extending to $J=8-7$. Predictably, the luminosity of the warm component rises slightly, from $7.9 \times 10^{6}$ to $9.7 \times 10^{6} \mathrm{~L}_{\odot}$, and the cold component luminosity is virtually unchanged (where the luminosities include the lines from $J=1-0$ to $8-7$ ). Conversely, the pressure in the warm component drops by about a factor of five and is more tightly constrained, indicating that with the extinction correction the gas is better represented by a single temperature component. Similarly, the cold component pressure also drops and is likewise more tightly constrained. The masses of the two components do not change significantly. Thus, the overall effect of the extinction correction is to raise the CO (warm component) luminosity by approximately $23 \%$, restrict the pressure likelihoods to smaller ranges (despite the additional uncertainties introduced by the uncertainties in the dust optical depths at the line frequencies), and leave the masses unchanged. For most of the remainder of the analysis, we consider the non-extinction corrected results because the properties of dust in the outflow are unknown and may not be similar to the general dense interstellar medium; however, we do describe the implications of making the extinction correction where they are relevant.

CARMA CO $J=1-0$ interferometry resolves the CVC into compact emission ("nucleus"), with a deconvolved radius of 60 pc, and an extended region ("envelope") at least twice that size (Alatalo et al 2011). We are unable to obtain fits of the CVC spectral line energy distribution that are consistent with the $J=1-0,2-1$, and $3-2$ lines if we restrict the emitting region to a radius of $60 \mathrm{pc}$ : the line fluxes are underproduced by the models and a substantially larger radius must be allowed, confirming the presence of the envelope. To attempt to quantify the fraction of low $-J$ emission arising in the nucleus and envelope, we ran models with the same percentages of $J=1-0,2-1$, and $3-2$ lines subtracted from the CVC fluxes and a compact source radius fixed at $60 \mathrm{pc}$, such that the fractions of the line fluxes subtracted represent the flux arising from the envelope. The 
model fits are not unique, but we conclude that likely at least $50 \%$ of the CVC flux from the $J=1-0$ and $J=2-1$ lines arises from the envelope 4

Only a single gas component is required to fit the Broad component spectral line energy distribution up to and including the $J=8-7$ line (Fig. [6 and Table 7): while the temperature is low (median value of $34 \mathrm{~K}$ ) and well constrained ( $\sigma$ range of $30 \mathrm{~K}$ to 41 ), the density likelihood distribution is very broad (partially because of the large uncertainties in the mid $-J$ Broad component line fluxes), ranging from $10^{4}$ to just over $10^{7} \mathrm{~cm}^{-3}$, leading to a broad pressure likelihood distribution $\left(3 \times 10^{5} \mathrm{~K} \mathrm{~cm}^{-3}\right.$ to $\left.1 \times 10^{9} \mathrm{~K} \mathrm{~cm}^{-3}\right)$. In fact, the density and pressure have uniform likelihood to the high (unphysical) end of the model grid, indicating unconstrained upper limits. Hence, while it seems likely that the gas is cold, its pressure and excitation are uncertain owing to the large allowed range in density. The mass of the Broad component is well constrained with $M_{\mathrm{Broad}} \sim 4 \times 10^{7} \mathrm{M}_{\odot}$, intermediate between the cold and warm CVC masses. Thus, the mass and luminosity $\left(6.9 \times 10^{6} \mathrm{~L}_{\odot}\right)$ of the Broad component are well constrained, but the gas pressure is not. As described next, using the full span of lines, including the higher-J, lines indicates both cold and warm components, with the cold component parameters being very similar to those derived using only the low and mid $-J$ lines.

In case (2) including the high- $J$ lines, the CVC $J=9-8$ to $J=13-12$ model line fluxes are subtracted from the FTS line fluxes to derive the high-J Broad component line fluxes. The Broad component now requires two temperature components to produce physical fits to the spectral line energy distribution (Fig. 7 and Table 7 ). 5 The spectral line

\footnotetext{
${ }^{4}$ It is unlikely that missing flux in the CARMA CO $J=1-0$ could substantially affect this comparison because Alatalo et al. (2011) measured very similar $J=1-0$ line fluxes with CARMA and the IRAM $30 \mathrm{~m}$.

${ }^{5} \mathrm{~A}$ single component yields solutions at low or high temperatures, depending on what
} 
energy distribution is shown in Fig. 8. With two components and the mass constraint, the luminosities of the cold and warm components are $L_{c} \sim 3.0 \times 10^{6} \mathrm{~L}_{\odot}$ and $L_{w} \sim 1.7 \times 10^{7} \mathrm{~L}_{\odot}$, and the temperatures are well separated, with maximum likelihood values of $\sim 30 \mathrm{~K}$ and $\sim 150 \mathrm{~K}$, and masses of approximately $\log M / M_{\odot}=7.6$ and 6.3 , respectively. Thus, the cold component dominates the mass and the warm component dominates the luminosity. The warm $\mathrm{H}_{2}$ density distribution is bimodal, with appreciable likelihood in the $10^{3.5}-10^{5}$ $\mathrm{cm}^{-3}$ range and above $10^{6} \mathrm{~cm}^{-3}$, but low likelihood between those ranges. The high range runs to the grid limit, but we disregard these densities $\left(n>10^{6} \mathrm{~cm}^{-3}\right)$ because they are unphysically high. The high pressure $\left(n T>10^{8} \mathrm{~K} \mathrm{~cm}^{-3}\right)$ are also unphysical because they correspond to the unphysical densities. While the extrapolation of the CVC to high-J introduces substantial uncertainty, the Broad component solutions are qualitatively similar to what has been observed for most other galaxies that have been studied in similar detail: two temperature components are required, the warm component is responsible for the bulk of the CO luminosity, and most of the mass is in the cold component. We reiterate that the warm Broad component parameters are meaningful only if our extrapolation of the separation into Broad and CVC components to the $J=9-8$ and higher lines, based on the case (1) model, is valid. However, the temperature of the cooler component, $\sim 60$ $\mathrm{K}$, is consistent with the analysis of the $J=8-7$ and below lines only. Applying the extinction correction drives up the $J=8-7$ and higher line fluxes and hence the warm Broad component luminosity to $3.5 \times 10^{7} L_{\odot}$, an increase of approximately 2 (where the luminosity now includes all of the lines from $J=1-0$ to $J=13-12$ ).

lines are included; removal of the mass constraint $M_{\text {warm }}<M_{\text {cold }}$ yields an insignificant warm component and extremely broad likelihood distributions for the cold component, which cannot alone account for a sufficiently broad range of physical conditions to fit the spectral line energy distribution. 
Our rotational CO molecular gas mass measurements can be compared to mass measurements from $\mathrm{H}_{2}$ observations. Roussel et al. (2007) used vibrational transitions of $\mathrm{H}_{2}$ to measure the molecular gas mass in the nuclear region of NGC1266 with an irregular 287 square arcsecond aperture. They measured gas masses of $1.3 \times 10^{7} \mathrm{M}_{\odot}$ and $2.2 \times 10^{6} \mathrm{M}_{\odot}$ in the temperature ranges $200 \mathrm{~K}<T<400 \mathrm{~K}$ and $400 \mathrm{~K}<T<1,450 \mathrm{~K}$, respectively. They did not have sufficient velocity resolution to separate the CVC and Broad components, both of which presumably contribute to their line fluxes. Using vibrational $\mathrm{H}_{2}$, they did not probe the gas in our cool component (probed with rotational transitions of CO). Their $200 \mathrm{~K}<T<400 \mathrm{~K}$ component overlaps substantially with our warm Broad component $\left(T=146_{-27}^{+974} \mathrm{~K}\right)$ and somewhat with our warm CVC component $\left(T=70_{-26}^{+130} \mathrm{~K}\right.$, where the error bars are $1 \sigma$ ), which yield a combined mass of $2.2 \times 10^{7} \mathrm{M}_{\odot}$, approximately $1.7 \times$ the $\mathrm{H}_{2}$ mass. These mass estimates compare favorably considering that much of our CVC warm component could be cooler than $200 \mathrm{~K}$. (The $\mathrm{T}>400 \mathrm{~K} \mathrm{H}_{2}$ component mass of $2.2 \times 10^{6} \mathrm{M}_{\odot}$ may also overlap with our Broad component, which has non-zero temperature likelihood up to $\sim 1,000 \mathrm{~K}$.) Thus, the $\mathrm{H}_{2}$ and CO-derived masses provide consistency checks, which the temperature estimates pass, but do not provide direct comparisons.

\section{Discussion}

NGC 1266 is notably complex for an S0 galaxy: there are at least five discernible components of $\mathrm{CO}$ emission from the nuclear region: (1) and (2) the CVC, presumably a disk, comprised of cool and warm components and with mass dominated by the cool component, but in which the warm component dominates the luminosity; (3) an extended,

cool CVC envelope of radius $r \gtrsim 100 \mathrm{pc}$; and (4) and (5) the cool (more massive) and warm (more luminous) Broad components associated with the outflow. In fact, more components (or a continuum of physical conditions) are likely present, but they cannot be distinguished 
with the data in hand. Here we compare estimates of the physical conditions of the various components, with a focus on masses, and discuss the gas excitation mechanisms.

\subsection{Physical Conditions of the CO Emission Components with a Focus on Masses}

Our modeling indicates cool and warm molecular gas masses for the CVC - including the envelope - of $M_{\mathrm{CVC}, c} \simeq 2 \times 10^{8} \mathrm{M}_{\odot}$ and $M_{\mathrm{CVC}, w}=2 \times 10^{7} \mathrm{M}_{\odot}$, so the CVC mass is dominated by the cold ( $\sim 10 \mathrm{~K})$ component. For comparison, Alatalo et al. (2011) used a CO conversion factor $\mathrm{X}_{\mathrm{CO}}=2 \times 10^{20} \mathrm{~cm}^{-2}\left(\mathrm{~K} \mathrm{~km} \mathrm{~s}^{-1}\right)^{-1}$ and peak CO $(\mathrm{J}=1-0)$ flux

of $24.3 \mathrm{Jy} \mathrm{km} \mathrm{s}^{-1}$ beam $^{-1}$ to estimate the molecular gas mass. They found a nuclear (i.e., excluding the envelope) CVC mass of $M_{\mathrm{CVC}, \text { nuc }} \approx 4 \times 10^{8} \mathrm{M}_{\odot}$ (the $\mathrm{X}_{\mathrm{CO}}$ factor should be relatively insensitive to the mass in the warm component). They use two additional methods to determine the CVC mass. Firstly, along the major axis in the position-velocity diagram they trace rotation out to a radius of $\sim 54 \mathrm{pc}$ and derive a maximum projected rotation velocity of $110 \mathrm{~km} \mathrm{~s}^{-1}$ (34inclination), from which they measure an enclosed dynamical nuclear mass of $M_{\mathrm{d} y n, n u c} \approx 5 \times 10^{8} \mathrm{M}_{\odot}$. Secondly, from an IRAM $30 \mathrm{~m}{ }^{13} \mathrm{CO}$ $(J=1-0)$ line flux and a relation between ${ }^{13} \mathrm{CO}$ line flux and extinction, they derive $M_{\mathrm{CVC}, \text { nuc }} \approx 3 \times 10^{8} \mathrm{M}_{\odot}$. By assuming that the rest of the CVC mass not in the nucleus is in the envelope, they estimate a mass of $M_{\mathrm{CVC} \text {,env }} \approx 6 \times 10^{8} \mathrm{M}_{\odot}$ in the envelope. Their combined nucleus and envelope mass of $M_{\mathrm{CVC}} \approx 10^{9} \mathrm{M}_{\odot}$, is just outside the $1 \sigma$ upper bound of our model; hence it cannot be considered inconsistent with our results. In fact, given the uncertainties, these values are all remarkably consistent. Our new observations and modeling have added to our understanding by showing that $10 \%$ of the CVC mass is in a warm, high pressure component $\left(n T=10^{6.3} \mathrm{~K} \mathrm{~cm}^{-3}\right.$ with a $\pm 1 \sigma$ range of approximately one dex). 
Estimation of the mass and physical conditions of the Broad component, the molecular outflow from the nucleus of NGC 1266, is important because it will yield insight into the acceleration of the outflow and because the gas could enrich the halo of NGC 1266. To summarize the physical conditions we derived for the Broad component: The low and mid $-J$ spectral line energy distribution is well fit by a single component $(T=30-40 \mathrm{~K}$, $\left.M \sim 4 \times 10^{7} \mathrm{M}_{\odot}, L \approx 6.9 \times 10^{6} \mathrm{~L}_{\odot}\right)$. Similarly, when the model CVC high- $J$ line fluxes are subtracted from the FTS line fluxes to yield Broad component high- $J$ line fluxes, both $\operatorname{warm}\left(L_{\mathrm{b} r o a d, w} \sim 2 \times 10^{7} \mathrm{~L}_{\odot}, P_{\mathrm{broad}, w} \sim 4 \times 10^{7} \mathrm{~K} \mathrm{~cm}^{-3}, M_{\mathrm{broad}, w} \sim 2 \times 10^{6} \mathrm{M}_{\odot}\right)$ and cool $\left(L_{\mathrm{b} \text { road,c }} \sim 3 \times 10^{6} \mathrm{~L}_{\odot}, M_{\mathrm{broad}, c} \sim 2 \times 10^{7} \mathrm{M}_{\odot}\right.$ to $\left.8 \times 10^{7} \mathrm{M}_{\odot}\right)$ Broad components are also required 6 . The low temperature component is subdominant in luminosity. Alatalo et al. (2011) also used RADEX modeling for the three lowest- $J$ CO lines, which showed (very non-uniquely) that the $J=1-0$ to $3-2$ line fluxes were consistent with $n \sim 10^{3} \mathrm{~cm}^{-3}$, $T \sim 100 \mathrm{~K}$ and $N(\mathrm{CO}) \sim 10^{16} \mathrm{~cm}^{-2}$, and an outflow mass of $2.4 \times 10^{7} \mathrm{M}_{\odot}$ for a $\mathrm{CO} / \mathrm{H}_{2}$ abundance of $1 \times 10^{-4}$. Using our assumed relative $\mathrm{CO}$ abundance of $3 \times 10^{-4}$ instead, this would yield a molecular gas mass of $8 \times 10^{6} \mathrm{M}_{\odot}$, which is not consistent with our result. Our mass estimate includes more lines and determines the parameter likelihoods rather than just finding a solution that fits the data. It is likely, therefore, that the mass of the outflow is approximately one order of magnitude smaller than the mass of the CVC.

In a recent paper, Alatalo et al. (2015) argue that the Broad component mass is $2 \times 10^{8} M_{\odot}$, nearly an order of magnitude larger than their previous estimate, with important implications for the driving of the outflow. This revised mass is based on the use of a CO conversion factor commonly used for ULIRGs (Bolatto, Wolfire, \& Leroy 2013). Alatalo et al. adopt this conversion factor on the grounds that the outflow must be optically thick in $\mathrm{CO}$ and dominated by dense gas, as they detect high-velocity components

\footnotetext{
${ }^{6}$ The luminosity is 2 times larger with the extinction correction applied.
} 
in CARMA observations of the $\mathrm{HCN} J=1-0$ (blueshifted and redshifted) and CS $J=2-1$ (blueshifted only) lines. This also raises their outflow mass estimate to $110 M_{\odot}$ $\mathrm{yr}^{-1}$. However, we are skeptical of this conclusion. Even if the broad line emission is both optically thick and arises in dense gas, that does not imply that the ULIRG conversion factor is appropriate for determining the gas mass from the CO line flux. Also, their revised outflow mass is comparable to our estimate for that of the CVC component, which would mean that the latter does not dominate the gas mass.

The dust mass (Table 5) also provides an estimate of the total mass of the molecular gas. Assuming a gas-to-dust mass ratio of 100 (K14 argue it could be up to a factor of two smaller), our dust mass converts to a gas mass of $2.2 \times 10^{8} \mathrm{M}_{\odot}$. Combined, the ensemble of measurements favors a total gas mass nearer to $10^{8}$ than $10^{9} \mathrm{M}_{\odot}$. We note that the primary uncertainty in our molecular gas estimates is the relative abundance of $\mathrm{CO}$ to $\mathrm{H}_{2}$, which likely contributes an uncertainty of approximately a factor of two to our molecular gas masses in addition to the statistical uncertainties in the tables and figures, and that the molecular gas masses may be biased low if anything because of our relative abundance choice $\left(3 \times 10^{-4}\right)$, but we are not subject to uncertainties in the $\mathrm{CO} \mathrm{X}_{\mathrm{CO}}$ factor or lack of knowledge of the $\mathrm{CO}$ rotational level excitation.

Alatalo et al. (2011) estimate a gas outflow rate of $13 \mathrm{M}_{\odot} \mathrm{yr}^{-1}$, corresponding to a depletion timescale of $\tau_{\text {out }}<85 \mathrm{Myr}$. The precision of our mass measurements significantly improve upon this estimate and suggest that the gas depletion time may be even shorter. Using the conversion from total infrared luminosity (8 to $1000 \mu \mathrm{m}$ ) to star formation rate of Bell (2003), if the far-infrared luminosity of NGC 1266 is due entirely to star formation its star formation rate is approximately $6 \mathrm{M}_{\odot} \mathrm{yr}^{-1}$. This corresponds to a gas depletion timescale $\left(\tau_{\mathrm{s} f}\right)$ approximately twice as long as $\tau_{\text {out }}$, suggesting that much of the reservoir will likely be ejected from the nuclear region before it can be converted to stars. 


\subsection{Gas Excitation Mechanisms}

The ratio of total $\mathrm{CO}$ to far-infrared luminosities, $L_{\mathrm{CO}} / L_{\mathrm{FIR}}$, is anomalously large, $1.7 \times 10^{-3}$, compared to the norm of $4 \times 10^{-4}$ found in the sample of K14 7 In that sample it is exceeded only by that of NGC 6240, a near-ULIRG merger with two nuclei, a buried AGN, and a strong case for shock-excited warm molecular gas (Meijerink et al. 2013). M82 and Arp 220, both with luminosities dominated by star formation, have CO spectral line energy distributions that peak around the $J=7-6$ line, then turn down for higher $J_{\text {upper }}$ (Fig. 9). In contrast, Mrk 231, a Seyfert 1 galaxy, has a CO spectral line energy distribution (characteristic of AGN dominated galaxies) that remains flat above $J=7-6$ for several lines before declining more slowly than starburst galaxies. NGC 1266's CO spectral line energy distribution is intermediate, suggesting perhaps that either both star formation and the AGN contribute to the CO excitation, or that the AGN is simply too weak to dominate the excitation of the high $-J$ lines.

Using the additional HIFI observations reported here, we are able to separate the CO luminosity of the Broad component from that of the CVC. For the CVC, the derived $L_{\mathrm{CO}} / L_{\mathrm{FIR}}=3 \times 10^{-4}$ is indistinguishable from the mean value in the K14 sample. This implies that this ratio must be substantially larger for the outflow, and possibly very large if the far-infrared emission is dominated by the CVC. This has important implications for the energetics of the outflow, as we discuss below.

There are multiple mechanisms that could contribute to the heating and excitation of the warm, Broad (outflow) component of the gas: photodissociation regions (PDRs)

\footnotetext{
${ }^{7} L_{\mathrm{CO}} / L_{\mathrm{FIR}}=1.7 \times 10^{-3}$ is extinction corrected, for comparison to the K14 result which is also extinction corrected. For NGC $1266, L_{\mathrm{CO}} / L_{\mathrm{FIR}}=1.0 \times 10^{-3}$ without an extinction correction.
} 
associated with high-mass star formation regions; shocks from outflows, infall, or other gas dynamics; cosmic rays; and an X-ray dissociation region (XDR) produced by the AGN. Given the modest star formation rate and sub-dominance of cosmic rays in the warm molecular gas heating of starburst galaxies (e.g., M 82: Panuzzo et al. 2010; Arp 220: Rangwala et al. 2011), cosmic rays are not a likely candidate (cosmic rays are still likely an important source of heating of the cold component of the CVC and even of the cold Broad [outflow] component, given the probable high optical depth of the clouds). Pellegrini et al. (2013) considered three excitation mechanisms for CO in NGC 1266: PDRs, XDRs, and shocks. With only mid and high $-J$ line fluxes from Herschel SPIRE FTS observations, which could not spectrally resolve the lines, they did not consider the CVC and Broad components separately. While a two-component PDR model $\left(n_{\mathrm{H}}=10^{3.75} \mathrm{~cm}^{-3}\right.$ and $\mathrm{G}_{0}=$ $\left.10^{6}\right)$ and $\left(n_{\mathrm{H}}=10^{5.5} \mathrm{~cm}^{-3}\right.$ and $\left.\mathrm{G}_{0}=10^{3.5}\right)$ could explain both the $\mathrm{CO}$ spectral line energy distribution and the rotation lines of $\mathrm{H}_{2}$ observed by Spitzer, Pellegrini et al. (2013) ruled this model out because it predicted a $L_{\mathrm{CO}} / L_{\mathrm{FIR}}$ ratio three orders of magnitude smaller than the observed ratio. XDRs were also ruled out based on the $L_{\mathrm{CO}} / L_{\mathrm{FIR}}$ ratio 8 Pellegrini et al. (2013) argued that the CO SLED was a composite, with the lowest- $J$ lines produced in a PDR and the higher $-J$ lines produced by C-shocks, which can also produce the observed water emission. Meijerink et al. (2013) reached similar conclusions about NGC 6240 from their analysis of the Herschel SPIRE FTS CO spectral line energy distribution.

\footnotetext{
${ }^{8}$ However, they misinterpreted Rangwala et al. (2011) to argue against XDRs. Quoting from their paper: "We do detect absorption from $\mathrm{H}_{2} \mathrm{O}^{+}$, but this has been seen in systems where XDR are ruled out (e.g., Arp220; Rangwala et al. 2011 )." In fact, an XDR is present in Arp 220 - it is required to explain the high column density of molecular ions; however, the AGN is not the primary heating mechanism for the molecular gas that is traced by the $\mathrm{CO}$ emission.
} 
Here we revisit the possibility of PDRs as an important agent in the heating of the warm molecular gas, using the additional information obtained by resolving the CVC and Broad components in the $J=1-0$ to $J=8-7$ lines with ground-based and HIFI observations. Specifically, we consider the $L_{\mathrm{CO}}(J=6-5) / L_{\mathrm{FIR}}, L_{\mathrm{CO}}(J=6-5) / L_{\mathrm{CO}}(J=1-0)$, $L_{\mathrm{CO}}(J=11-10) / L_{\mathrm{CO}}(J=6-5)$, and $L_{\mathrm{CO}}(J=11-10) / L_{\mathrm{CO}}(J=1-0)$ ratios, combined with the number density constraints from our non-LTE modeling. The PDR models are those of Wolfire et al. (2010) (M. Wolfire, private communication 2011).

The results are shown in Figure 10. Consider first the $L_{\mathrm{CO}}(J=6-5) / L_{\mathrm{FIR}}$ ratio (Figure 10 upper left). The green and blue curves show the observed values for the CVC and Broad components, respectively, while the dashed vertical lines show the allowed density regimes, similarly color-coded. The CVC and Broad line-to-continuum ratios are nearly identical, reflecting the similarity of the fluxes in the two components. For both the warm CVC and Broad components, we find that PDR solutions are acceptable for $G_{0}$ between $10^{3}$ and $10^{4}$. However, there is an important caveat to this conclusion. In the absence of high-resolution spectral and continuum imaging, it is not known what fraction of the far-infrared luminosity should be assigned to each component. The observed ratios have therefore been calculated by assigning $100 \%$ of the far-infrared flux to both. It is highly likely that this results in a significant underestimate of the true line-to-continuum ratio for the Broad component. As discussed above, the total $L_{\mathrm{CO}} / L_{\mathrm{FIR}}$ ratio for the CVC component is typical for star-forming galaxies (K14) when all of the far-infrared luminosity is assigned to this component, while for the Broad component this ratio is unusually high. Combined with the other evidence for star formation in this galaxy (Alatalo et al. 2011; Alatalo et al. 2014), this suggests that a large fraction of the far-IR arises from the CVC component. If only a fraction $f_{B}$ should be associated with the Broad component emission, then the acceptable $\log G_{0}$ range in Figure 10a will shift down by approximately $\log \left(1 / f_{B}\right)$ (for $f_{B} \gtrsim 3 \%$ ), implying that $G_{0}<10^{3}$ for $f_{B}<10 \%$. This has important implications 
when we consider the other PDR diagnostics.

Figure 10 upper right compares the $L_{\mathrm{CO}}(J=6-5) / L_{\mathrm{CO}}(J=1-0)$ ratios for the two components to the PDR model predictions. We still find acceptable solutions for $G_{0}$ in the $10^{3}-10^{4}$ range; for the CVC, the PDR models are pushed to the low end of the density range, while for the Broad component acceptable solutions for both diagnostics require $G_{0} \sim 10^{4}$ and densities at the upper end of the range allowed by the likelihood analysis.

The line ratios plotted in the remaining two figure panels must be regarded as uncertain, since the division of the $J=11-10$ line flux into CVC and Broad components relies on the extrapolation of the CVC model spectrum to lines for which we have only unresolved FTS fluxes. However, the constraints imposed by the $L_{\mathrm{CO}}(J=11-10) / L_{\mathrm{CO}}(J=6-5)$, and $L_{\mathrm{CO}}(J=11-10) / L_{\mathrm{CO}}(J=1-0)$ ratios are similar, for both components. For the CVC emission, the solutions imposed by matching the $J=11-10$ line ratios are somewhat at odds with the other PDR constraints: although a similar range of $G_{0}$ is allowed, satisfying the high $-J$ line ratios require densities that are much larger (an order of magnitude or more) than for the line-to-continuum and $L_{\mathrm{CO}}(J=6-5) / L_{\mathrm{CO}}(J=1-0)$ ratios. The CVC $J=11$ - 10 line flux would have to be much smaller than our extrapolated value to vitiate this conclusion. For the Broad component, the density constraints can be simultaneously satisfied for all the observed ratios, but the high $-J$ line ratios require $G_{0} \gtrsim 10^{4}$. If $f_{B} \ll 1$, as seems likely, then there would be no PDR model parameter space in which all of the constraints can be met. As with the CVC component, there will be acceptable solutions if the Broad $J=11-10$ line flux is much smaller than our estimate, but if this is true, then this rules out a PDR origin for the CVC component, as it must produce the remainder of the line flux. Thus, independent of our modeling, at least one of the two components cannot be explained as PDR emission.

In summary, the mid $-J$ and low $-J$ lines from both the CVC and Broad components 
can in principle arise in PDRs, provided $G_{0} \lesssim 10^{4}$. However, the higher $-J$ emission, as represented by the $J=11-10$ line, does not fit easily into this picture, and at least one of the two components must contain line contributions from a non-PDR source, independent of our decomposition of the $J=11-10$ line flux. We therefore conclude that shocks are likely a substantial and possibly dominant heating mechanism for the warm molecular gas in the CVC and Broad components. This seems particularly likely for the Broad component, given the identification of this component with the molecular outflow.

X-ray dissociation regions (XDRs) have been found to be the likely excitation source of warm nuclear CO emission in galaxies with AGN (e.g., Meijerink et al. 2013). Is it possible that the AGN in NGC1266 powers the CO emission via an XDR? A definitive analysis of $\mathrm{CO}$ emission from an XDR is not possible because the molecular gas densities and temperatures are not constrained tightly enough and because the AGN X-ray luminosity is uncertain. While it is possible that the $L_{\mathrm{CO}} / L_{\mathrm{FIR}}\left(L_{\mathrm{CO}} / L_{\mathrm{Bolometric}}=1 \times 10^{-5}\right.$ and $2.9 \times 10^{-4}$ for the CVC cold and warm components, respectively, and $L_{\mathrm{CO}} / L_{\text {Bolometric }}=1.1 \times 10^{-4}$ and $6.3 \times 10^{-4}$ for the Broad cold and warm components, respectively, not extinction corrected) could be accommodated by an XDR (see Bradford et al. 2009), a substantial fraction of $\mathrm{CO}$ emission arising from an XDR seems unlikely because (a) the AGN would have to dominate the bolometric luminosity of the galaxy, for which there is presently no observational support, and (b) the conversion of hard X-ray luminosity to CO luminosity would have to be almost maximally efficient $(\sim 1 \%)$.

\section{Conclusions}

New Herschel Space Observatory HIFI data enable us to spectroscopically resolve the nuclear central velocity component (CVC; $123 \mathrm{~km} \mathrm{~s}^{-1}$ FWHM) and Broad (outflow; 278 $\mathrm{km} \mathrm{s}^{-1} \mathrm{FWHM}$ ) components of CO $J=5-4, J=6-5, J=7-6$, and $J=8-7$ lines for 
the first time. Modeling of the $J=1-0$ to $J=8-7$ CVC reveals two components: cold $(T \sim 10 \mathrm{~K})$, comprising most of the mass $\left(2 \times 10^{8} \mathrm{M}_{\odot}\right)$ and a minority of the luminosity $(3 \%)$, and warm $(T \sim 100 \mathrm{~K})$, with a minority of the mass $\left(2 \times 10^{7} \mathrm{M}_{\odot}\right)$ and a majority of the luminosity (97\%). The CVC $L_{\mathrm{CO}} / L_{\mathrm{FIR}}$ is typical of star-forming galaxies and consistent with a modest star formation rate of $6 \mathrm{M}_{\odot} \mathrm{yr}^{-1}$. With an estimated outflow rate of 13 $\mathrm{M}_{\odot} \mathrm{yr}^{-1}$ the reservoir would be depleted before it could be converted to stars at the current consumption rates, but assuming that the CVC feeds the outflow perhaps $1 / 3$ of the mass is destined to form stars. Our models fail to fit the CVC CO spectral line energy distribution in the lowest few lines if the radius is $<60 \mathrm{pc}$, hence we conclude that on order $50 \%$ of the emission arises from a larger region, consistent with the previous conclusions of Alatalo et al. (2011).

For the Broad component identified as an outflow, as for the CVC, the gas densities and pressures were not well constrained, partially because of the relatively large number of temperature components and uncertain velocity decomposition for the high-J lines. Subtraction of the CVC J $=9-8$ and above model line fluxes from Herschel SPIRE FTS line fluxes to estimate the Broad component line fluxes yields strong evidence that it also has warm and cold components, with again nearly an order of magnitude more CO luminosity arising from the warm component and masses of $4 \times 10^{7} M_{\odot}$ (cold) and $2 \times 10^{6} M_{\odot}($ warm $)$. Unlike the CVC, the outflow $L_{\mathrm{CO}} / L_{\mathrm{FIR}}$ ratio is anomalously high Extinction corrections under the assumption of well-mixed dust and molecular gas raise the outflow luminosity as much as a factor of 2 but do not have a strong effect on the derived masses.

Comparison of the CO spectral line energy distribution, far-infrared luminosity, and CO modeling results to photodissociation models indicate that the warm components of both the CVC and outflow cannot be excited by PDRs; while just one of them could be, 
it is likely that both warm components are shock excited (especially the outflow). Thus, our analysis provides strong evidence for the warm outflow gas (which is responsible for the bulk of the CO luminosity and anomalously high $L_{\mathrm{CO}} / L_{\mathrm{FIR}}$ ratio) being shock excited and driven by the AGN since the star formation is too anemic.

NGC 1266 remains enigmatic because the nuclear gas, with an apparently short lifetime because of the outflow and star formation, does not have a clear origin. High angular resolution dust continuum interferometry will be required to further clarify the molecular gas physical conditions and various components, although large-scale mapping (several 100 pc) may be required to further elucidate the origin of the molecular gas.

We thank the anonymous referee for a thoughtful and constructive report. We also thank Alex Conley for helpful discussions of modeling. SPIRE has been developed by a consortium of institutes led by Cardiff Univ. (UK) and including Univ. Lethbridge (Canada); NAOC (China); CEA, LAM (France); IFSI, Univ. Padua (Italy); IAC (Spain); Stockholm Observatory (Sweden); Imperial College London, RAL, UCL-MSSL, UKATC, Univ. Sussex (UK); Caltech, JPL, NHSC, Univ. Colorado (USA). This development has been supported by national funding agencies: CSA (Canada); NAOC (China); CEA, CNES, CNRS (France); ASI (Italy); MCINN (Spain); SNSB (Sweden); STFC, UKSA (UK); and NASA (USA).This research has made use of the NASA/IPAC Extragalactic Database (NED) which is operated by the Jet Propulsion Laboratory, California Institute of Technology, under contract with the National Aeronautics and Space Administration. This work is supported in part by NASA award 1432844. JRK gratefully acknowledges the support of an NSF Graduate Fellowship.

Facilities: Herschel Space Observatory 


\section{REFERENCES}

Alatalo, K, Lacy, M., Lanz, L, Bitsakis, T., Appleton, P. N., Nyland, K., Cales, S., Chang, P., Davis, T. A., de Zeeuw, P. T., Lonsdale, C. J., Martín, S., Meier, D. S., \& Ogle, P. M. 2015, ApJ, 798, 31

Alatalo, K., Nyland, K., Graves, G., Deustua, S., Shapiro Griffin, K., Duc, P.-A., Cappellari, M., McDermid, R. M.., Davis, T.A.., Crocker, A.F., Young, L.M., Chang, P., Scott, N., Cales, S.L., Bayet, E., Blitz, L., Bois, M., Bournaud, F., Bureau, M., Davies, R. L., de Zeeuw, P.T., Emsellem, E., Khochfar, S., Krajnović, D., Kuntschner, H., Morganti, R., Naab, T., Oosterloo, T., Sarzi, M., Serra, P., \& Weijmans, A.-M. 2014, ApJ, 780, 186

Alatalo, K., Blitz, L., Young, L. M., Davis, T. A., Bureau, M., Lopez, L. A., Cappellari, M., Scott, N., Shapiro, K. L., Crocker, A. F., Martín, S., Bois, M., Bournaud, F., Davies, R. L., de Zeeuw, P. T., Duc, P.-A., Emsellem, E., Falcón-Barroso, J., Khochfar, S., Krajnović, D., Kuntschner, H., Lablanche, P.-Y., McDermid, R. M., Morganti, R., Naab, T., Oosterloo, T., Sarzi, M., Serra, P., \& Weijmans, A. 2011, ApJ, 735, 88

Bell, E. F. 2003, ApJ, 586, 794

Bolatto, A. D., Wolfire, M., \& Leroy, A. K. 2013, ARA\&A, 51, 207

Bradford, C.M., Aguirre, J.E., Aikin, R., Bock, J.J., Earle, L., Glenn, J., Inami, H., Maloney, P.R., Matsuhara, H., Naylor, B.J., Nguyen, H.T., \& Zmuidzinas, J. 2009, ApJ, 705, 112

Brauher, J.R., Dale, D. A., \& Helou, G. 2008, ApJS, 178, 280

Buchner, J., Georgakakis, A., Nandra, K., Hsu, L., Rangel, C., Brightman, M., Merloni, A., Salvato, M., Donley, J., and Kocevski, D. 2014, A\&A, 564, A125 
Cappellari, M., Emsellem, E., Krajnović, D., McDermid, R. M., Scott, N., Verdoes Kleijn, G. A., Young, L. M.., Alatalo, K., Bacon, R., Blitz, L., Bois, M., Bournaud, F., Bureau, M., Davies, R. L., Davis, T. A., de Zeeuw, P. T., Duc, P.-A., Khochfar, S., Kuntschner, H., Lablanche, P.-Y., Morganti, R., Naab, T., Oosterloo, T., Sarzi, M., Serra, P., \& Weijmans, A.-M. 2011, MNRAS, 413, 813

Casey, C.M. 2012, MNRAS, 425, 3094

Dale, D. A., Gil de Paz, A., Gordon, K. D., et al. 2007, ApJ, 655, 863

Davis, T. A.; Krajnović, D., McDermid, R. M., Bureau, M., Sarzi, M., Nyland, K., Alatalo, K., Bayet, E., Blitz, L., Bois, M., Bournaud, F., Cappellari, M., Crocker, A., Davies, R.L., de Zeeuw, P.T., Duc, P.-A., Emsellem, E., Khochfar, S., Kuntschner, H., Lablanche, P.-Y., Morganti, R., Naab, T., Oosterloo, T., Scott, N., Serra, P., Weijmans, A.-M., \& Young, L.M. 2012, MNRAS, 426, 1574

Dunne, L., Eales, S. A., \& Edmunds, M. G. 2003, MNRAS, 341, 589

de Graauw, Th., Helmich, F. P., Phillips, T. G. et al. 2010, A\&A, 518, L6

Feroz, F., \& Hobson, M. P. 2008, MNRAS, 384, 449

Feroz, F., Hobson, M. P., \& Bridges, M. 2009, MNRAS, 398, 1601

Feroz, F., Hobson, M. P., Cameron, E., \& Pettit, A.N. 2013, arXiv:1306.2144

Griffin, M.J., Abergel, A., Abreu, A., et al. 2010, A\&A, 518, L3

(K14) Kamenetzky, J., Rangwala, N., Glenn, J., Maloney, P.R., \& Conley, A. 2014, ApJ, 795,174 
Kamenetzky, J., Glenn, J., Rangwala, N., Maloney, P., Bradford, M., Wilson, C. D., Bendo, G. J., Baes, M., Boselli, A., Cooray, A., Isaak, K. G., Lebouteiller, V., Madden, S., Panuzzo, P., Schirm, M. R. P., Spinoglio, L., \& Wu, R. 2012, ApJ, 753, 70

Lacy, J. H., Knacke, R., Geballe, T. R., \& Tokuna, A. T. 1994, ApJ, 428, 69

Meijerink, R., Kristensen, L. E., Weiß, A., van der Werf, P. P., Walter, F., Spaans, M., Loenen, A. F., Fischer, J., Israel, F. P., Isaak, K., Papadopoulos, P. P., Aalto, S., Armus, L., Charmandaris, V., Dasyra, K. M., Diaz-Santos, T., Evans, A., Gao, Y., González-Alfonso, E., Güsten, R., Henkel, C., Kramer, C., Lord, S., Martín-Pintado, J., Naylor, D., Sanders, D. B., Smith, H., Spinoglio, L., Stacey, G., Veilleux, S., \& Wiedner, M. C. 2013, ApJ, 762, L16

Nyland, K., Alatalo, K., Wrobel, J. M., Young, L. M., Morganti, R., Davis, T. A., de Zeeuw, P. T., Deustua, S., \& Bureau, M. 2013, ApJ, 779, 173

Panuzzo, P., Rangwala, N., Rykala, A., et al. 2010, A\&A, 518, L37

Pellegrini, E. W., Smith, J. D., Wolfire, M. G., Draine, B. T., Crocker, A. F., Croxall, K. V., van der Werf, P., Dale, D. A., Rigopoulou, D., Wilson, C. D., Schinnerer, E., Groves, B. A., Kreckel, K., Sandstrom, K. M., Armus, L., Calzetti, D., Murphy, E. J., Walter, F., Koda, J., Bayet, E., Beirao, P., Bolatto, A. D., Bradford, M., Brinks, E., Hunt, L., Kennicutt, R., Knapen, J. H., Leroy, A. K., Rosolowsky, E., Vigroux, L., \& Hopwood, R. H. B. 2013, ApJ, 779, L19

Pilbratt, G. L., Riedinger, J. R., Passvogel, T., Crone, G., Doyle, D., Gageur, U., Heras, A. M., Jewell, C., Metcalfe, L., Ott, S., \& Schmidt, M. 2010, A\&A, 518, L1

Rangwala, N., Maloney, P. R., Glenn, J., Wilson, C. D., Rykala, A., Isaak, K., Baes, M., Bendo, G. J., Boselli, A., Bradford, C. M., Clements, D. L., Cooray, A., Fulton, T., 
Imhof, P., Kamenetzky, J., Madden, S. C., Mentuch, E., Sacchi, N., Sauvage, M., Schirm, M. R. P., Smith, M. W. L., Spinoglio, L., \& Wolfire, M. 2011, ApJ, 743, 94

Roussel, H., Helou, G., Hollenbach, D. J., Draine, B. T., Smith, J. D., Armus, L., Schinnerer, E., Walter, F., Engelbracht, C. W., Thornley, M. D., Kennicutt, R. C., Calzetti, D., Dale, D. A., Murphy, E. J., \& Bot, C. 2007, ApJ, 669, 959

Sanders, D. B., Mazzarella, J. M., Kim, D., et al. 2003, AJ, 126, 1607

Scott, K.S., Lupu, R. E., Aguirre, J. E., et al. 2011, ApJ, 733, 29

Temi, P., Brighenti, F., \& Mathews, W.G. 2009, ApJ, 707, 890

van der Tak, F. F. S., Black, J. H., Schöier, F. L., Jansen, D. J., \& van Dishoeck, E. F. 2007, A\&A, 468, 627

Wolfire, M.G., Hollenbach, D., \& McKee, C. F. 2010, ApJ, 716, 1191 


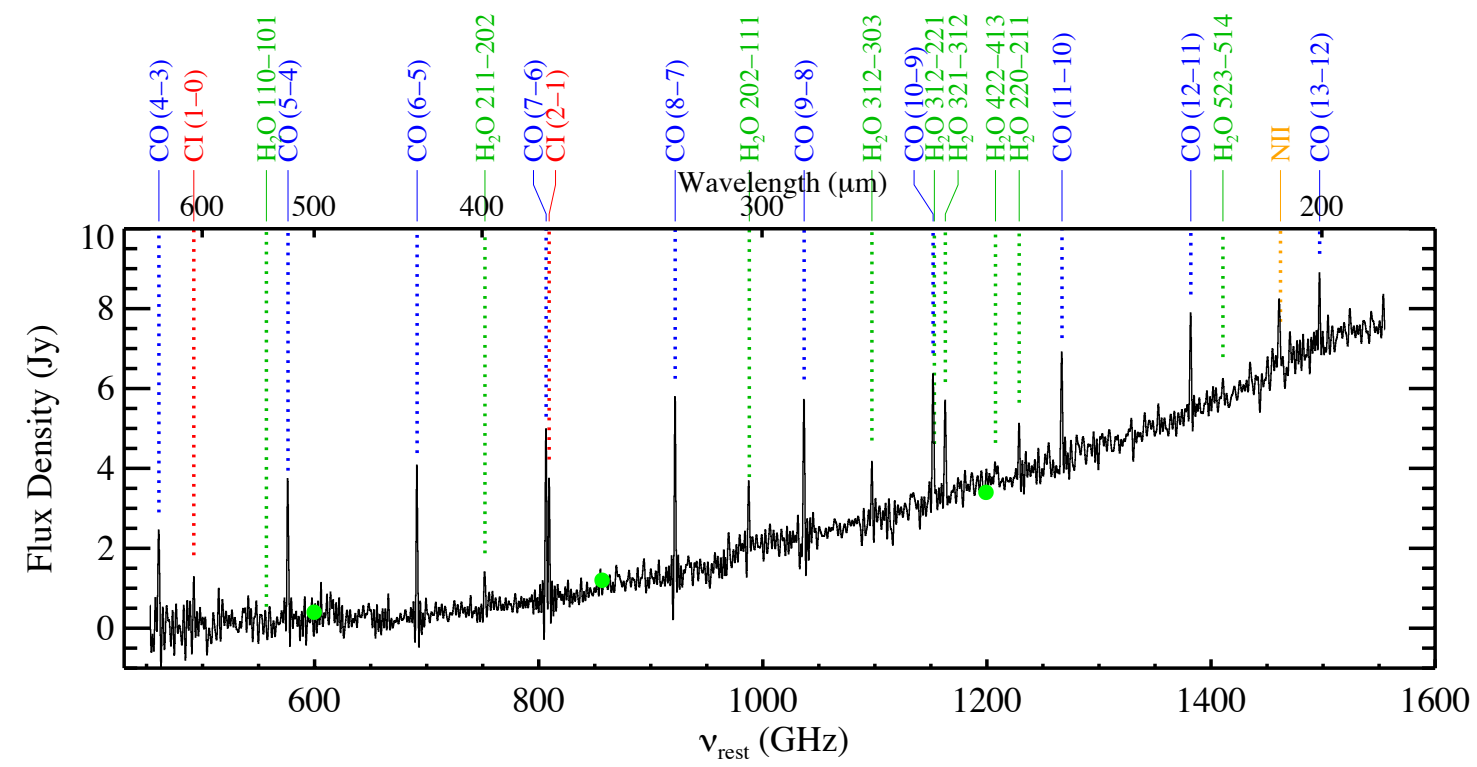

Fig. 1.- Combined Herschel SPIRE FTS Spectrometer Short-Wave and Spectrometer LongWave spectra. The dust continuum is apparent rising to higher frequencies and prominent emission lines are labeled. Filled green circles at approximately $600 \mathrm{GHz}, 860 \mathrm{GHz}$, and $1220 \mathrm{GHz}$ indicate the photometry of SPIRE PLW, PMW, and PSW, which are consistent with the spectroscopic flux densities. 

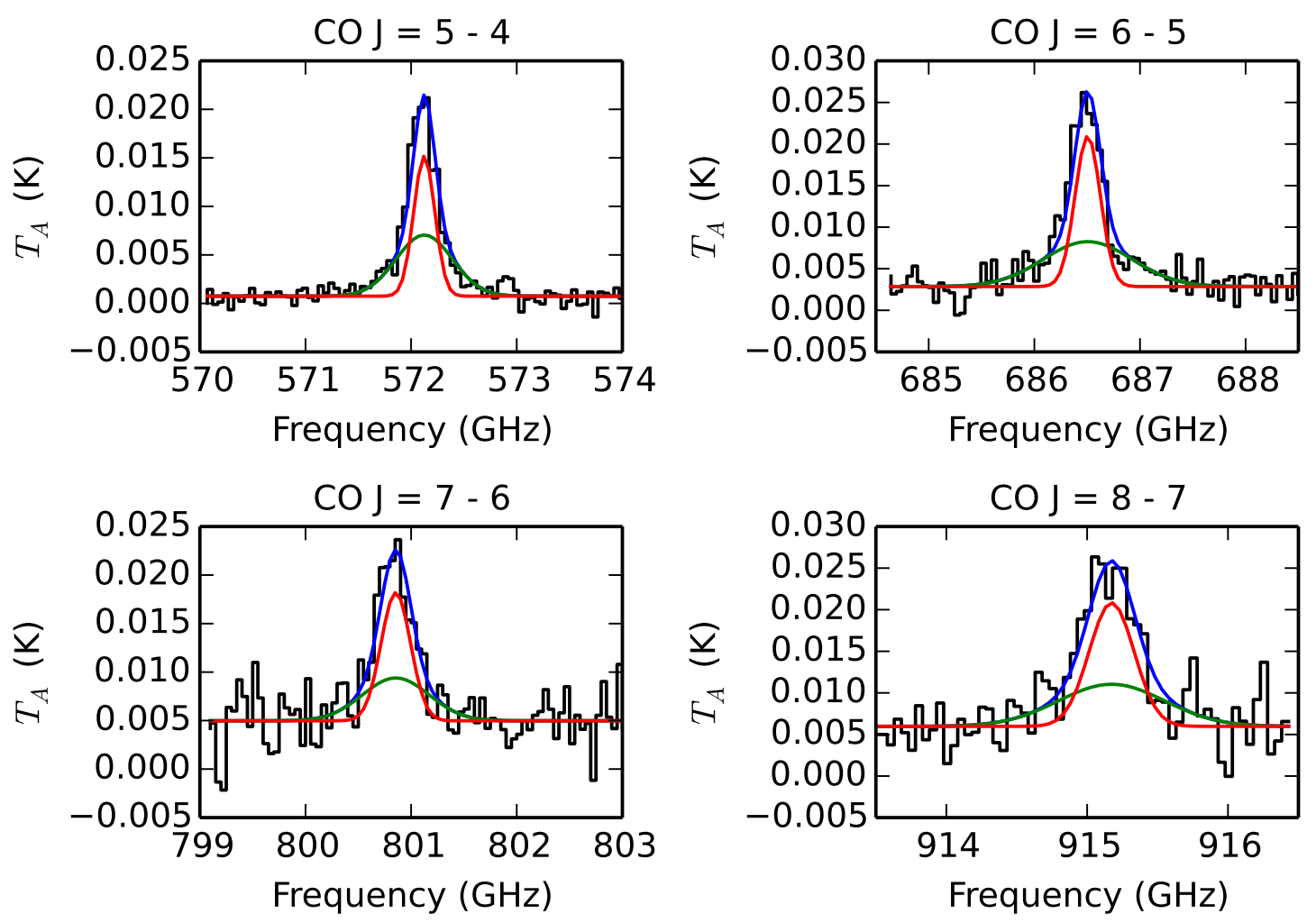

Fig. 2.- Herschel HIFI CO spectra and their decompositions into CVC (red) and Broad (outflow, green) components. The blue lines are their sums. 


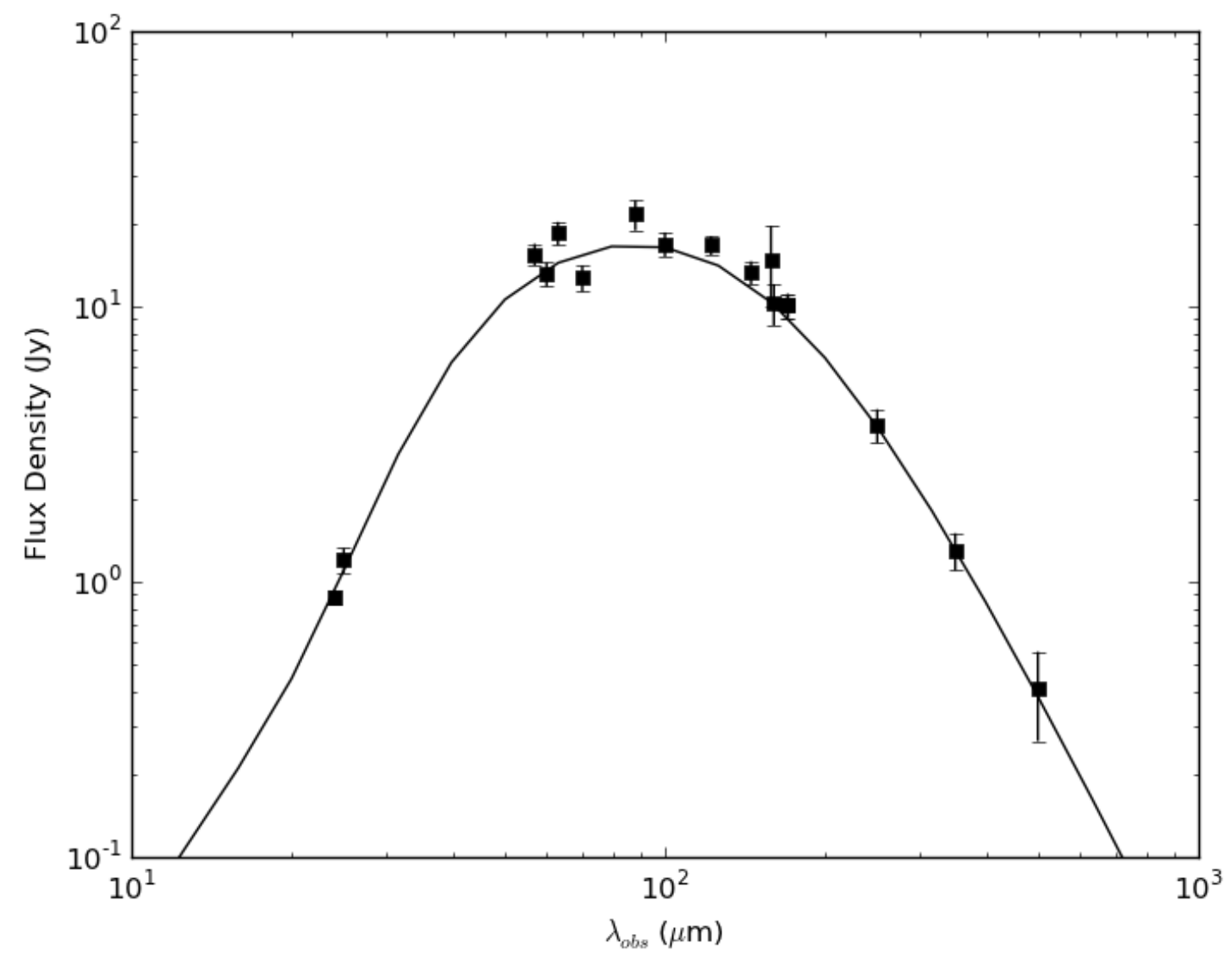

Fig. 3.- Continuum photometry (listed in Table 4) and best-fit dust spectrum. 

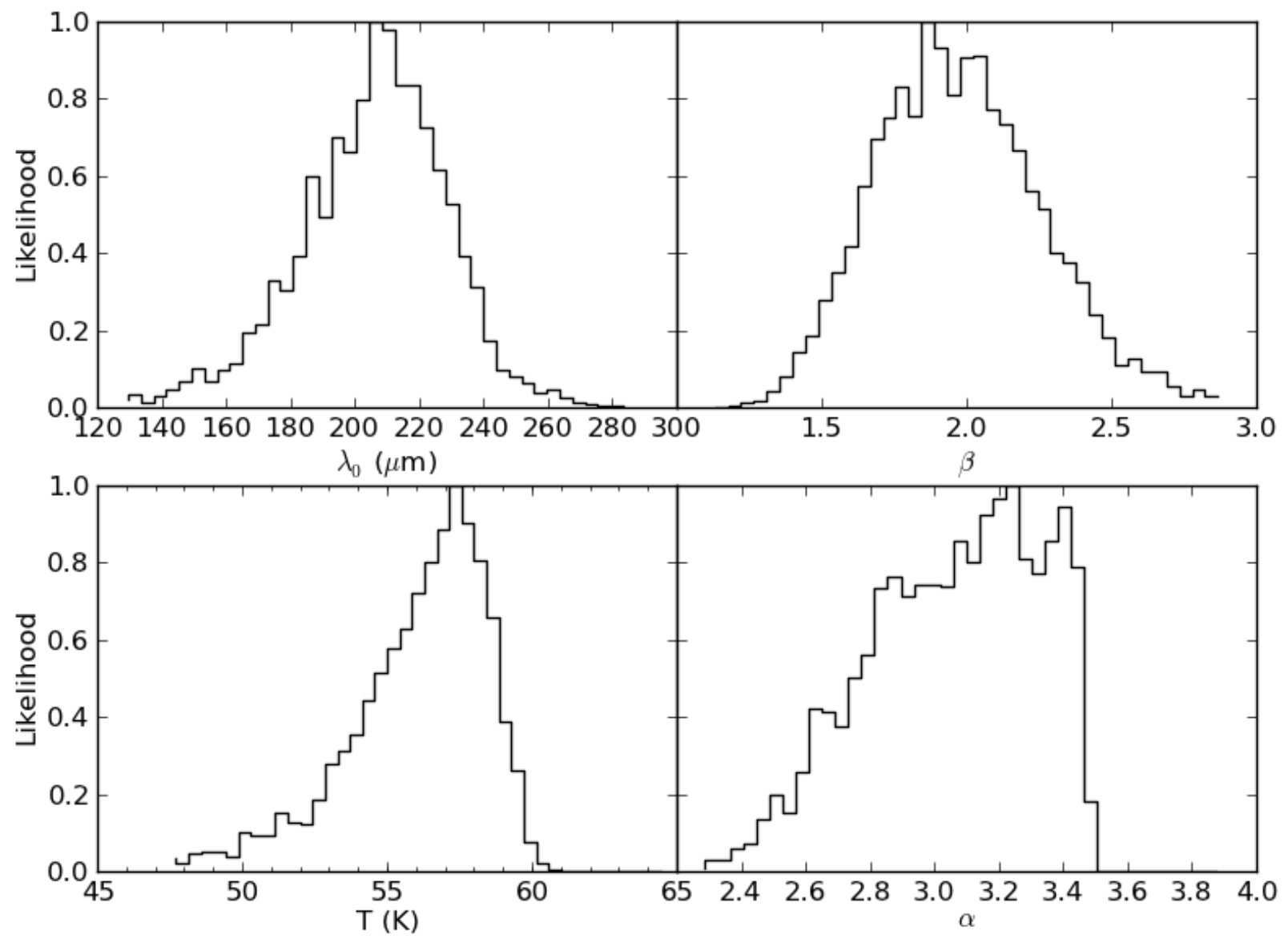

Fig. 4.- Best-fit dust parameter likelihood distributions: $\lambda_{0}$, wavelength at which the optical depth is unity, $\beta$, spectral index of emissivity, temperature $T$, and Wien-side spectral index $\alpha$. 

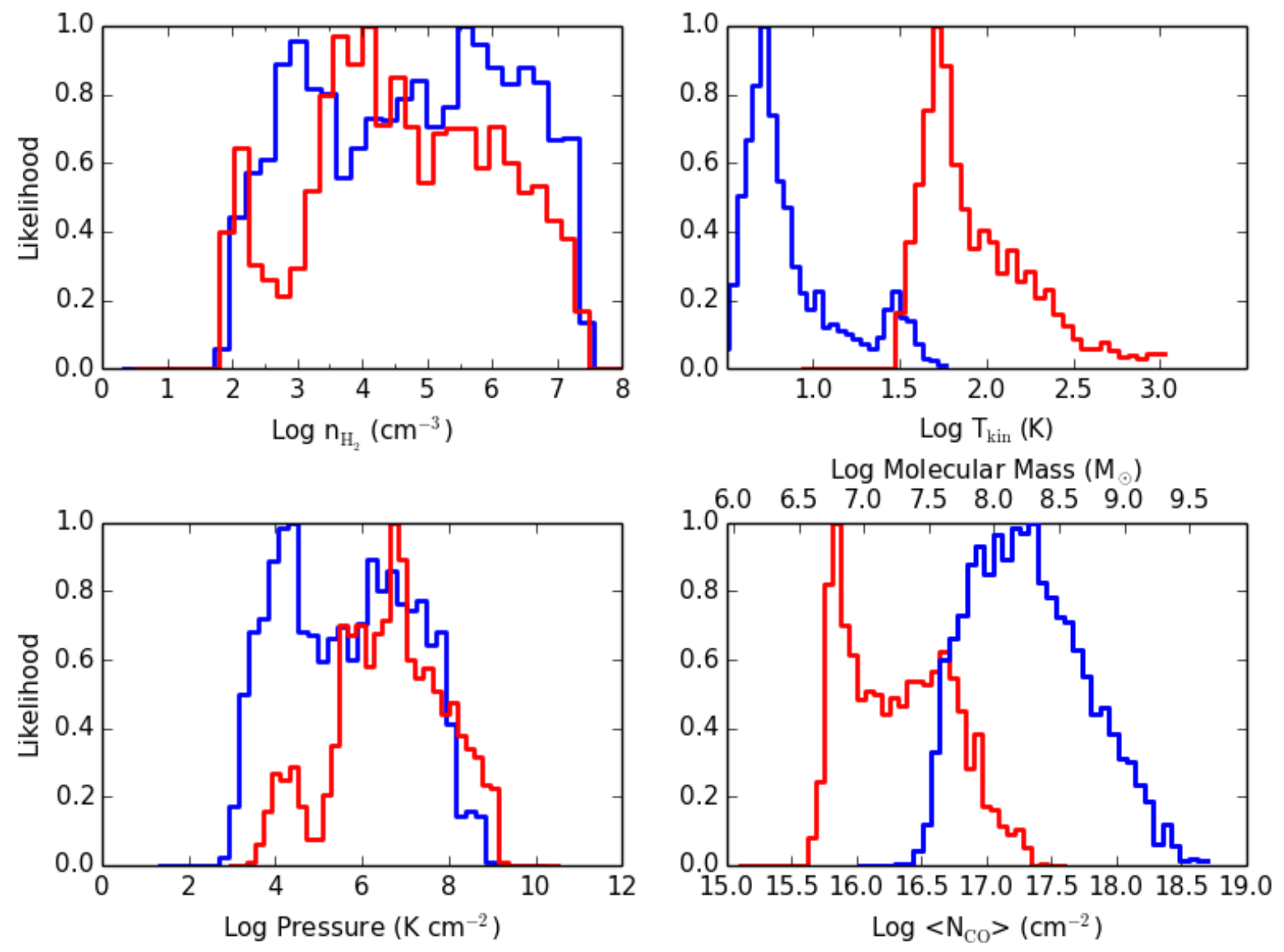

Fig. 5.- Marginalized parameter likelihoods for the CVC cold (blue) and warm (red) components, subject to the constraint that $\mathrm{M}_{\text {warm }}<\mathrm{M}_{\text {cold }}$ and using $\mathrm{CO}$ lines from $J=1-0$ to $J=8-7$. 

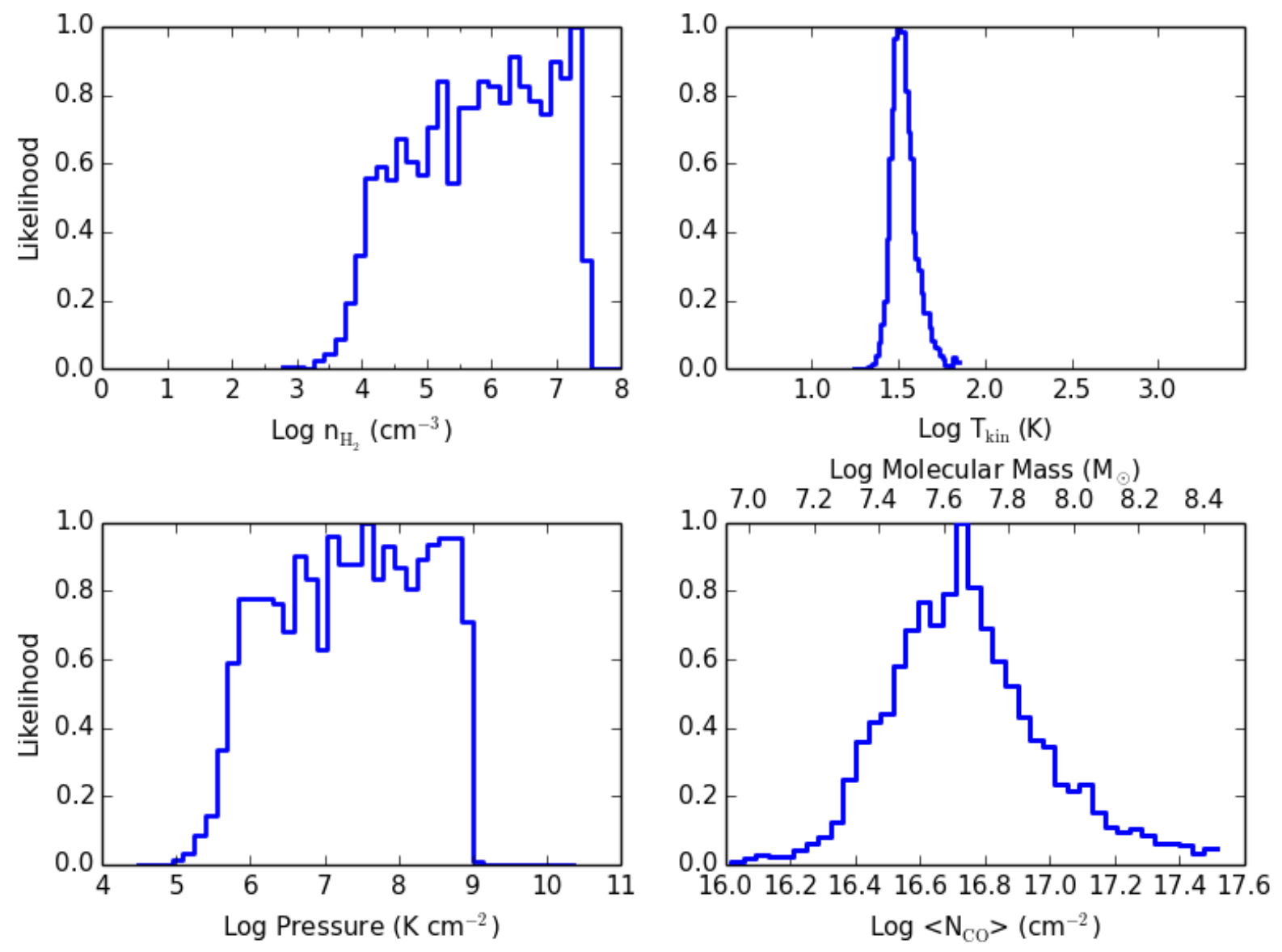

Fig. 6.- Marginalized parameter likelihoods for the Broad component using CO lines from $J=1-0$ to $J=8-7$. Only one component is required to fit the Broad component spectral line energy distribution without an extinction correction or higher-J lines. 

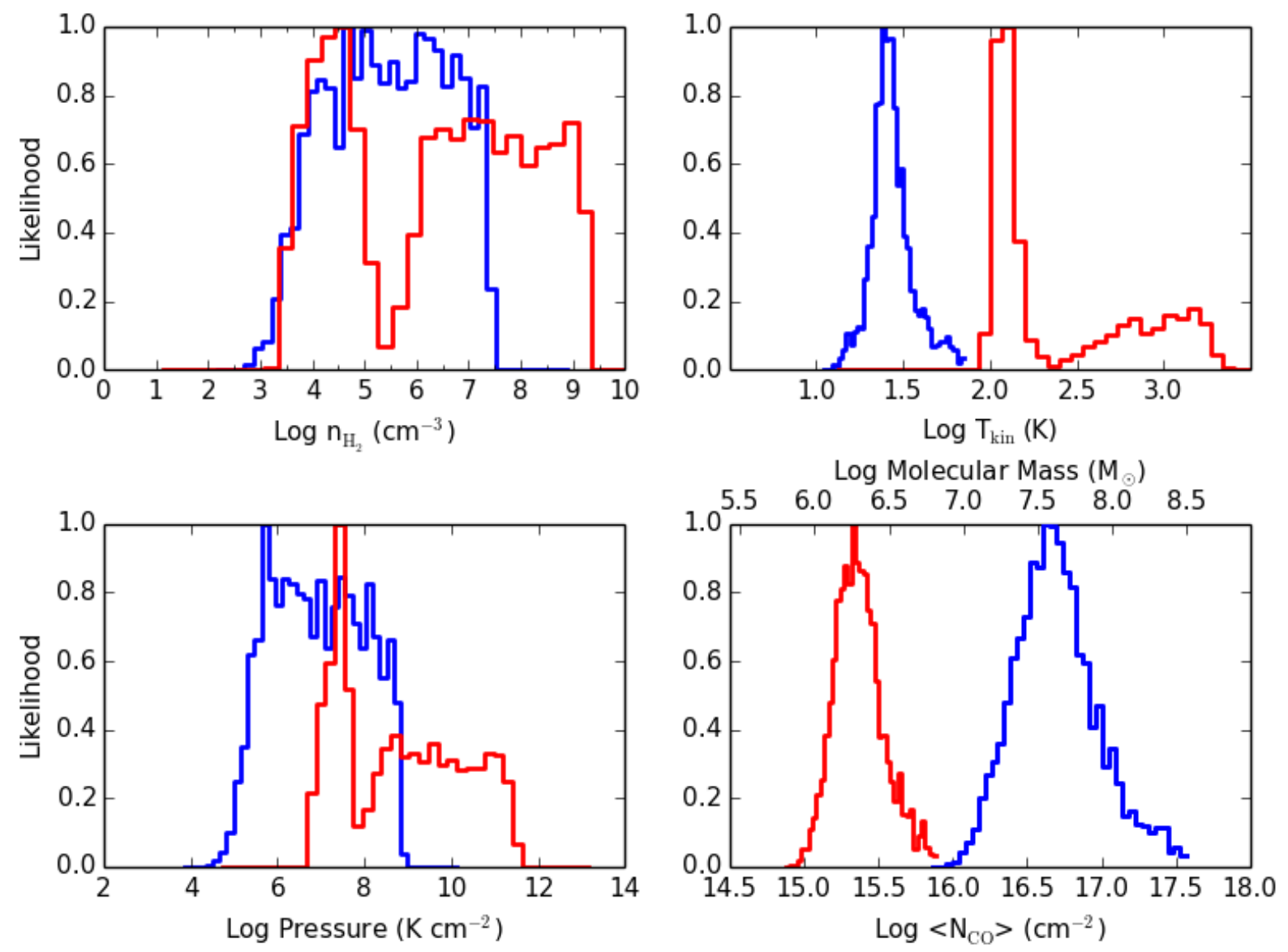

Fig. 7.- Marginalized parameter likelihoods for the Broad component including the $J=$ $9-8$ to $J=13-12$ lines, where the model CVC line fluxes are subtracted from the FTS line fluxes. These models are subject to the constraint that $\mathrm{M}_{\text {warm }}<\mathrm{M}_{\text {cold }}$. The cold component is blue and the warm component is red. 


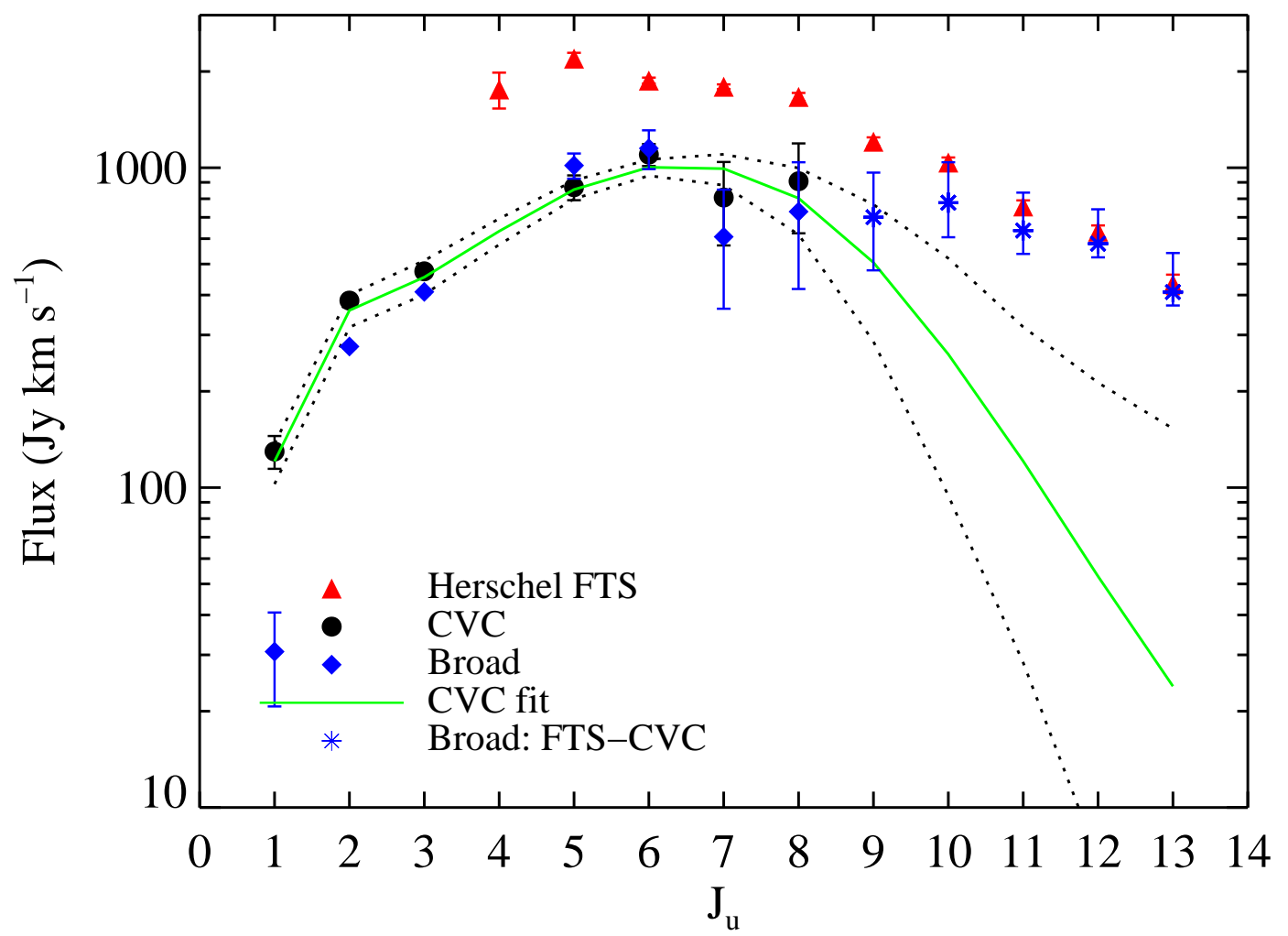

Fig. 8.- (Non extinction-corrected) CO spectral line energy distribution, including Herschel FTS, Herschel HIFI, and ground-based observations from Alatalo et al. (2011). "Broad" (blue diamonds) refers to the line fluxes from Gaussian profile fits. The Broad component lines above $J=8-7$ ("Broad: FTS-CVC", blue asterisks) were derived by subtracting the CVC fit (green line and black dotted $\pm 1 \sigma$ lines) from the FTS line fluxes (red triangles), incorporating the line flux likelihoods to derive Broad component line flux error bars. All error bars are $1 \sigma$. 


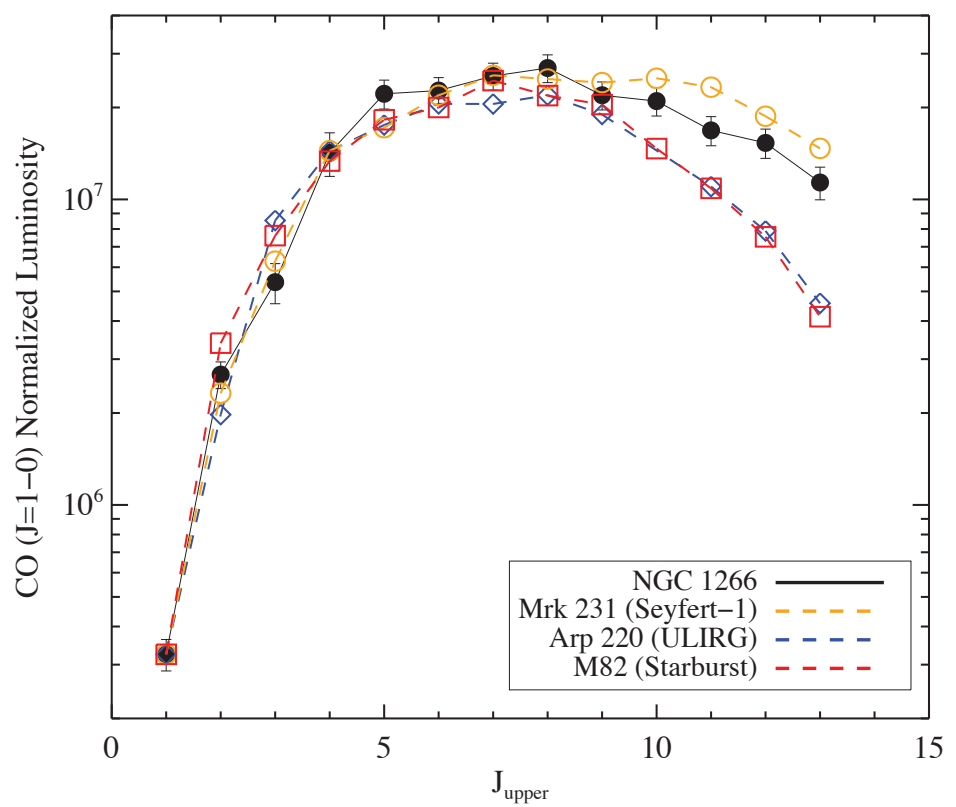

Fig. 9.- A comparison of the CO spectral line energy distributions of Mk231 (Seyfert galaxy), NGC1266, and Arp 220 and M82, which are galaxies whose far-infrared luminosities are dominated by star formation. The spectral line energy distributions are normalized at $J=6-5$. 

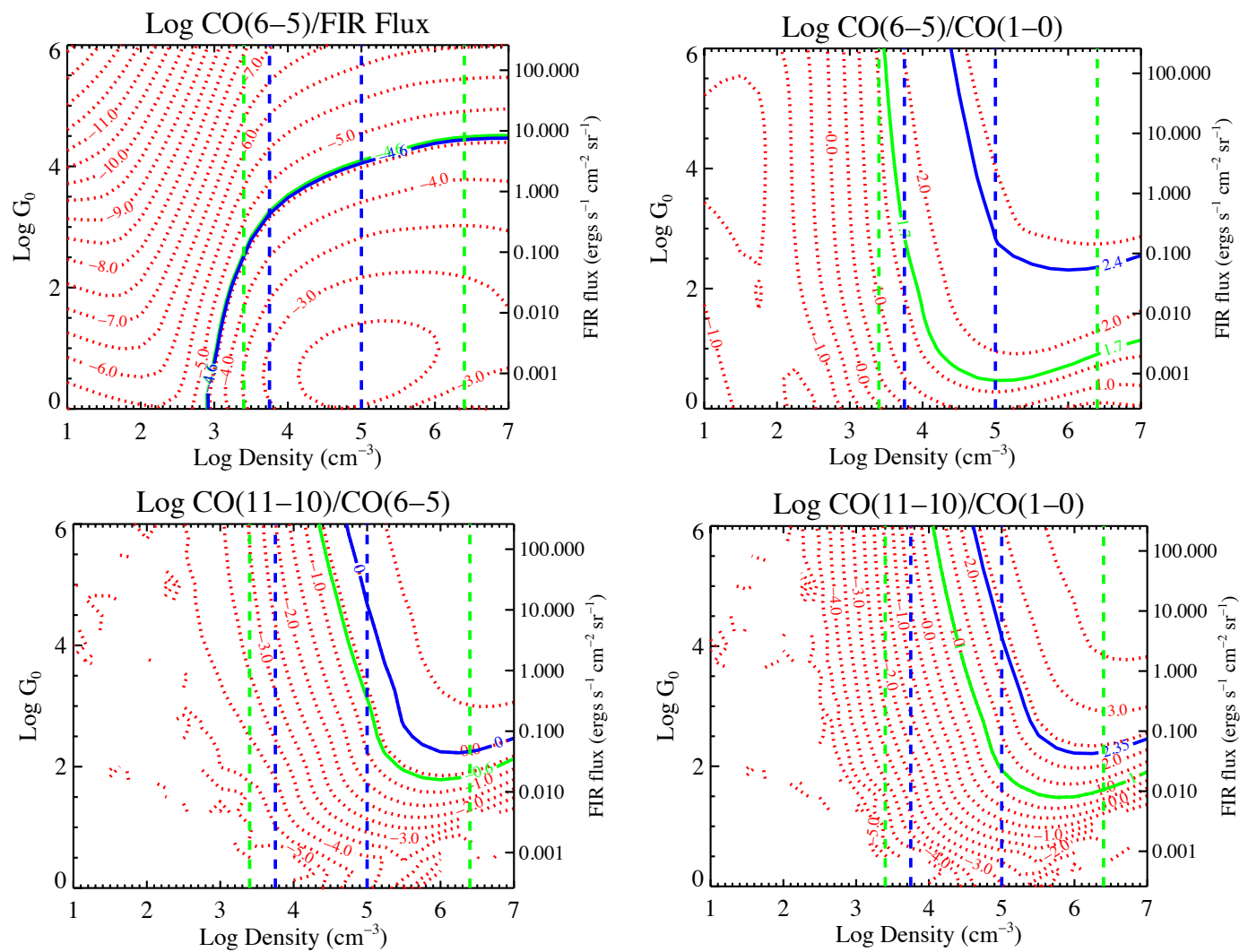

Fig. 10. - PDR model grid, with gas density along the x-axis and UV radiation intensity parameter $G_{0}$ on the y-axis, overplotted with the observed ratios. The models are those of Wolfire et al (2010). (a) The ratio of the CO $J=6-5$ line flux to the far-infrared line flux. The ratio observed for the CVC is in green, that for the Broad component is in blue. The green (blue) vertical dashed lines show the density ranges allowed by the likelihood models ( $1 \sigma$ for the CVC and $3 \sigma$ for the Broad component). The observed ratios have been calculated by assigning $100 \%$ of the far-infrared flux to each component separately; see text for discussion. The right-hand vertical scale shows the model far-infrared surface brightness corresponding to the values of $G_{0}$. (b) As in (a), but for the $L_{\mathrm{CO}}(J=6-5) / L_{\mathrm{CO}}(J=1-0)$ line ratio. (c) As in (a), but for the $L_{\mathrm{CO}}(J=11-10) / L_{\mathrm{CO}}(J=6-5)$ line ratio. Division of the $J=11-10$ line into CVC and Broad components is done using the best-fitting likelihood model; see text. (d) As in (c), but for the $L_{\mathrm{CO}}(J=11-10) / L_{\mathrm{CO}}(J=1-0)$ line ratio. 
Table 1. Herschel Observations

\begin{tabular}{clcrc}
\hline \hline Observation & Instrument/Band & OBSID & OD & $\begin{array}{c}\text { tintegration } \\
\text { (s) }\end{array}$ \\
\hline Lines \& Continuum & SPIRE-FTS & 1342239353 & 1012 & 5640 \\
$\mathrm{CO} J=5-4$ & HIFI 1b & 1342238647 & 996 & 4063 \\
$\mathrm{CO} J=6-5$ & HIFI 2a & 1342238179 & 985 & 3918 \\
$\mathrm{CO} J=7-6$ & HIFI 3a & 1342239585 & 1016 & 3945 \\
$\mathrm{CO} J=8-7$ & HIFI 3b & 1342237619 & 980 & 3928 \\
\hline
\end{tabular}


Table 2. Herschel SPIRE-FTS Line Fluxes

\begin{tabular}{|c|c|c|c|c|}
\hline ID & Transition & $\begin{array}{l}\text { Rest Frequency } \\
\qquad(\mathrm{GHz})\end{array}$ & $\begin{array}{c}\text { Flux } \\
\left(\mathrm{Jy} \mathrm{km} \mathrm{s}^{-1}\right)\end{array}$ & $\begin{array}{c}\sigma \\
\left(\mathrm{Jy} \mathrm{km} \mathrm{s}{ }^{-1}\right)\end{array}$ \\
\hline CI & $J=1-0$ & 492.16064 & 789.4 & 135.3 \\
\hline CI & $J=2-1$ & 809.34198 & 1139.6 & 29.2 \\
\hline${ }^{12} \mathrm{CO}$ & $J=4-3$ & 461.04077 & 1757.2 & 225.4 \\
\hline${ }^{12} \mathrm{CO}$ & $J=5-4$ & 576.26794 & 2193.6 & 93.2 \\
\hline${ }^{12} \mathrm{CO}$ & $J=6-5$ & 691.47308 & 1873.3 & 40.1 \\
\hline${ }^{12} \mathrm{CO}$ & $J=7-6$ & 806.65179 & 1792.4 & 29.9 \\
\hline${ }^{12} \mathrm{CO}$ & $J=8-7$ & 921.79968 & 1665.4 & 48.0 \\
\hline${ }^{12} \mathrm{CO}$ & $J=9-8$ & 1036.91235 & 1206.0 & 38.4 \\
\hline${ }^{12} \mathrm{CO}$ & $J=10-9$ & 1151.98547 & 1038.6 & 38.2 \\
\hline${ }^{12} \mathrm{CO}$ & $J=11-10$ & 1267.01453 & 757.2 & 33.3 \\
\hline${ }^{12} \mathrm{CO}$ & $J=12-11$ & 1381.99512 & 631.7 & 28.6 \\
\hline${ }^{12} \mathrm{CO}$ & $J=13-12$ & 1496.92285 & 432.7 & 30.4 \\
\hline NII & ${ }^{3} P_{1}-{ }^{3} P_{0}$ & 1461.1319 & 750.2 & 82.6 \\
\hline $\mathrm{H}_{2} \mathrm{O}$ & $2_{11}-2_{02}$ & 752.0332 & 430.4 & 28.9 \\
\hline $\mathrm{H}_{2} \mathrm{O}$ & $2_{02}-1_{11}$ & 987.92676 & 628.8 & 55.5 \\
\hline $\mathrm{H}_{2} \mathrm{O}$ & $3_{12}-3_{03}$ & 1097.36475 & 462.6 & 35.7 \\
\hline $\mathrm{H}_{2} \mathrm{O}$ & $3_{21}-3_{12}$ & 1162.91162 & 761.9 & 33.3 \\
\hline $\mathrm{H}_{2} \mathrm{O}$ & $4_{22}-4_{13}$ & 1207.63867 & 147.7 & 33.5 \\
\hline $\mathrm{H}_{2} \mathrm{O}$ & $2_{20}-2_{11}$ & 1228.78882 & 372.4 & 34.4 \\
\hline $\mathrm{H}_{2} \mathrm{O}$ & $5_{23}-5_{14}$ & 1410.61804 & 102.3 & 24.5 \\
\hline
\end{tabular}


Table 3. CO Velocity Component Fluxes

\begin{tabular}{lccc}
\hline \hline & & & \\
Transition & CVC Flux & Broad Component Flux & Broad Component Width \\
& $\left(\mathrm{Jy} \mathrm{km} \mathrm{s}^{-1}\right)$ & $\left(\mathrm{Jy} \mathrm{km} \mathrm{s}^{-1}\right)$ & $\left(\mathrm{km} \mathrm{s}^{-1}\right)$ \\
\hline $\mathrm{J}=1-0$ & $130 \pm 3$ & $31 \pm 10$ & $597 \pm 108$ \\
$\mathrm{~J}=2-1$ & $384 \pm 6$ & $276 \pm 4$ & $283 \pm 5$ \\
$\mathrm{~J}=3-2$ & $474 \pm 9$ & $409 \pm 6$ & $274 \pm 5$ \\
$\mathrm{~J}=5-4$ & $870 \pm 80$ & $1020 \pm 90$ & $328 \pm 35$ \\
$\mathrm{~J}=6-5$ & $1100 \pm 90$ & $1150 \pm 160$ & $273 \pm 68$ \\
$\mathrm{~J}=7-6$ & $810 \pm 240$ & $610 \pm 250$ & $205 \pm 145$ \\
$\mathrm{~J}=8-7$ & $910 \pm 280$ & $730 \pm 310$ & \\
\hline
\end{tabular}

Note. - Uncertainties are $1 \sigma$. The CVC line widths were fixed at $123 \mathrm{~km} \mathrm{~s}^{-1}$ (see text). 
Table 4. Continuum Photometry

\begin{tabular}{|c|c|c|c|c|c|}
\hline $\begin{array}{l}\text { Wavelength } \\
\qquad(\mu \mathrm{m})\end{array}$ & $\begin{array}{c}\text { Flux Density } \\
(\mathrm{Jy})\end{array}$ & $\begin{array}{l}\sigma_{\text {stat }} \\
(\mathrm{Jy})\end{array}$ & $\begin{array}{c}\sigma_{\text {calib }} \\
(\mathrm{Jy})\end{array}$ & Facility & Ref. \\
\hline 250 & 3.690 & 0.185 & 0.185 & SPIRE-PSW & 1 \\
\hline 350 & 1.260 & 0.063 & 0.063 & SPIRE-PMW & 1 \\
\hline 500 & 0.370 & 0.019 & 0.019 & SPIRE-PLW & 1 \\
\hline 57 & 15.500 & 0.310 & 1.365 & ISO & 2 \\
\hline 63 & 18.500 & 0.370 & 1.762 & ISO & 2 \\
\hline 88 & 21.600 & 0.432 & 2.665 & ISO & 2 \\
\hline 122 & 16.700 & 0.334 & 1.256 & ISO & 2 \\
\hline 145 & 13.300 & 0.266 & 1.272 & ISO & 2 \\
\hline 158 & 14.800 & 0.296 & 4.791 & $I S O$ & 2 \\
\hline 170 & 10.100 & 0.202 & 0.979 & ISO & 2 \\
\hline 25 & 1.200 & 0.032 & 0.120 & $I R A S$ & 3 \\
\hline 60 & 13.130 & 0.045 & 1.313 & $I R A S$ & 3 \\
\hline 100 & 16.890 & 0.185 & 1.689 & $I R A S$ & 3 \\
\hline 24 & 0.843 & 0.008 & 0.034 & MIPS & 4,5 \\
\hline 70 & 10.304 & 0.118 & 0.515 & MIPS & 4,5 \\
\hline 160 & 6.339 & 0.144 & 0.761 & MIPS & 4,5 \\
\hline
\end{tabular}

References. - (1) This paper. (2) ISO: Brauher et al. (2008). (3) IRAS: Sanders et al. (2003). (4) and (5) Dale et al. (2007) and Temi et al. (2009), respectively. 
Table 5. Dust Continuum Fit

\begin{tabular}{cc}
\hline \hline Parameter & Mean $\pm 1 \sigma$ \\
\hline$T_{\text {dust }}(\mathrm{K})$ & $56 \pm 3$ \\
$\lambda_{0}(\mu \mathrm{m})$ & $206 \pm 25$ \\
$\beta$ & $2.00 \pm 0.29$ \\
$\alpha$ & $3.1 \pm 0.3$ \\
$\tau(100 \mu \mathrm{m})$ & $4.7 \pm 1.9$ \\
$\log \left[L_{\mathrm{FIR}}\left(\mathrm{L}_{\odot}, 8-1000 \mu \mathrm{m}\right)\right]$ & $10.44 \pm 0.01$ \\
$\log \left[M_{\text {dust }}\left(\mathrm{M}_{\odot}\right)\right]$ & $6.34 \pm 0.04$ \\
\hline
\end{tabular}

Note. - The dust mass uncertainty is statistical only: it does not account for the uncertainty in the dust opacity, which could be a factor of two. 
Table 6. CVC Gas Parameters Constrained by Models

\begin{tabular}{lcc}
\hline \hline \multicolumn{1}{c}{ Parameter } & Cold Comp Median $\pm 1 \sigma$ & Warm Comp Median $\pm 1 \sigma$ \\
\hline & & \\
$T_{k i n}(\mathrm{~K})$ & $6_{-2}^{+10}$ & $74_{-26}^{+130}$ \\
$\log _{10}\left(\left\langle N_{C O}\right\rangle_{\Omega}\right)$ & $17.3_{-0.4}^{+0.5}$ & $16.3_{-0.5}^{+0.5}$ \\
$\log _{10}($ Mass $)\left(\mathrm{M}_{\odot}\right)$ & $8.3_{-0.4}^{+0.5}$ & $7.3_{-0.5}^{+0.5}$ \\
$\log _{10}($ Luminosity $)\left(\mathrm{L}_{\odot}\right)$ & $5.44_{-0.18}^{+0.22}$ & $6.90_{-0.16}^{+0.16}$ \\
\hline
\end{tabular}


Table 7. Broad Component Gas Parameters Constrained by Models

\begin{tabular}{lcc}
\hline \hline \multicolumn{1}{c}{ Parameter } & First Comp Median $\pm 1 \sigma$ & Second Comp Median $\pm 1 \sigma$ \\
\hline & & \\
$T_{\text {kin }}(\mathrm{K})$ & & \\
$\log _{10}\left(\left\langle N_{C O}\right\rangle_{\Omega}\right)$ & $36_{-4}^{+5}$ & $\ldots$ \\
$\log _{10}($ Mass $)\left(\mathrm{M}_{\odot}\right)$ & $16.7_{-0.2}^{+0.2}$ & $\ldots$ \\
$\log _{10}($ Luminosity $)\left(\mathrm{L}_{\odot}\right)$ & $7.6_{-0.2}^{+0.2}$ & $\ldots$ \\
& $6.84_{-0.08}^{+0.06}$ & $\ldots$ \\
All lines & & \\
$T_{\text {kin }}(\mathrm{K})$ & & \\
$\log _{10}\left(\left\langle N_{C O}\right\rangle_{\Omega}\right)$ & $27_{-5}^{+9}$ & $146_{-27}^{+974}$ \\
$\log _{10}($ Mass $)\left(\mathrm{M}_{\odot}\right)$ & $16.7_{-0.3}^{+0.3}$ & $15.4_{-0.1}^{+0.2}$ \\
$\log _{10}($ Luminosity $)\left(\mathrm{L}_{\odot}\right)$ & $7.6_{-0.3}^{+0.3}$ & $6.3_{-0.1}^{+0.2}$ \\
\hline
\end{tabular}

